\title{
Enrichment in a General Class of Stoichiometric Producer-Consumer Population Growth Models
}

\author{
Harlan Stecha, c, Bruce Peckham a and John Pastor ${ }^{b}$ \\ a Department of Mathematics and Statistics, University of Minnesota Duluth \\ b Department of Biology, University of Minnesota Duluth \\ ${ }^{c}$ Communicating author: Department of Mathematics and Statistics, University of \\ Minnesota Duluth, Duluth, Minnesota 55812. Email: $\underline{\text { hstech@d.umn.edu }}$
}

Revision: December 20, 2011

Keywords: Producer-consumer models, stoichiometry, energy, nutrient enrichment.

\begin{abstract}
This paper presents the derivation and partial analysis of a general producer-consumer model. The model is stoichometric in that it includes the growth constraints imposed by species-specific biomass carbon to nutrient ratios. The model unifies the approaches of other studies in recent years, and is calibrated from an extensive review of the algae-Daphnia literature. Numerical simulations and bifurcation analysis are used to examine the impact of energy enrichment under nutrient and stoichiometric constraints. Our results suggest that the variety of system responses previously cited for related models can be attributed to the size of the total system nutrient pool, which is here assumed fixed. New, more complicated bifurcation sequences, such as multiple homoclinic bifurcations, are demonstrated as well. The mechanistic basis of the model permits us to show the robustness of the system's dynamics subject to alternate approaches to modeling producer and consumer biomass production.
\end{abstract}

1. Introduction. Over the last decade, significant progress has been made in the study of stoichiometric producer-consumer population models. Such models recognize that biomass creation and conversion processes depend simultaneously on both the carbon and nutrient requirements of the species involved. Early work focused on producer-consumer competition for a limited nutrient resource and the impact of nutrient levels on producer growth. See, for example, [36], [37], [18], [16]. However, these studies did not consider systems in which consumer biomass production depends on the variable nutrient levels of the producer population. This latter point was explicitly addressed in the work of Andersen [2] and Hessen \& Bjerkeng [20]. Subsequent studies have modeled open or closed systems, systems with multiple nutrient constraints, alternate methods for modeling the many subprocesses affecting consumer and producer growth, respiration and mortality, as well as the dynamic properties of systems with multiple producer or consumer populations.

There is a much smaller literature regarding the effects of energy enrichment on producer-consumer systems. For non-stoichiometric models (which only track the flow of carbon in the system), system enrichment is often assumed synonymous to an 
increase in the carrying capacity of the bottom-level species. For example, see [1]. However, in stoichiometric models enrichment must be modeled more mechanistically in order to distinguish between (say) increasing ambient light and increasing total system nutrient levels. While both such increases affect the primary productivity and the resulting consumer-free carrying capacity of the base-level producer community, their impact on higher trophic levels is more complex. Specifically, energy enrichment results in additional carbon resources for the consumer population, but in closed-nutrient systems may reduce the nutrient richness of the consumer's food source, hence consumer productivity. In contrast, nutrient enrichment increases a resource that producer and consumer populations sequester to different degrees, based on their different stoichiometric ratios.

The theoretical work of Rosenzweig [40] pointed out that enriching available energy/carbon for the producer can destabilize a system's coexistence state. He considers the variation of the classical Lotka-Volterra model that assumes Holling IIlike saturation of the consumer's foraging rate. In such non-stoichiometric models, once the coexistence state is destabilized, the system typically supports a unique, attracting, periodic coexistence state, whose amplitude of oscillation increases with energy enrichment.

Loladze, Kuang \& Elser [31] introduced stoichiometric effects to the usual realization of the Rosenzweig-MacArthur model [41]. They assumed that the system is closed (ie., possesses a fixed total nutrient budget) for a single nutrient that is partitioned between the producer and consumer populations. The nutrient concentration of the producer population is assumed flexible, with higher nutrient levels increasing both producer productivity and consumer biomass conversion efficiency. Their model is parameterized for a generic algae-Daphnia system with phosphorous the limiting nutrient. Numerical simulations of this ("LKE") model indicated that with increasing energy enrichment, a stable, producer monoculture gives way to a stable coexistence equilibrium state. With further enrichment this coexistence equilibrium can lose stability, resulting in a stable, attracting, periodic coexistence state. However, in contrast to the Rosenzweig-MacArthur model, the periodic state grows until energy enrichment has attained a critical level, at which point the periodic state is lost. This loss is coincident with the creation of a pair of new steady coexistence states - one stable and one unstable. With further energy enrichment, the consumer level for the attracting coexistence state declines ultimately to extinction, leaving only a stable producer monoculture for high energy levels. Consumer extinction apparently results if energy enrichment raises the producer carbon levels and thereby dilutes the nutrient pool to the point that the food quality $(\mathrm{N}: \mathrm{C})$ that the producer offers is too low for the consumer to sustain itself. Similar behavior is observed in an alternate, more process-based producer-consumer model [26], in close variants of the LKE model [12], and extensions to two-producer [30] and two-consumer [32] systems.

Subsequent to the LKE study, Diehl [8] presented a more mechanistic model of a similar algae-Daphnia system. While this model shares many of the same assumptions of the previous models, its form differs in that producer productivity, 
nutrient uptake, and biomass creation are founded on process-based sub-models. In fact, producer light interception is modeled to sufficient precision to allow the distinction between energy enrichment via increases in ambient light levels, decreases in water turbidity, and a reduction of water column depth. System parameterizations are similar to that of LKE, with the exception of the total nutrient levels, which are in Diehl's work reduced to levels that are more realistic of pelagic systems.

Numerical simulations of the Diehl model indicate that with increasing energy enrichment the system sustains a producer monoculture, then a steady coexistence equilibrium state which destabilizes to a stable periodic coexistence state. However, in contrast to the LKE model, further light enrichment eventually reduces the amplitude of coexistence oscillations. Under sufficiently high light enrichment these oscillations further decline and the coexistence steady state regains stability. Thus, the LKE and Diehl models differ significantly in their responses to energy enrichment, a point that Diehl attributed to his more mechanistic approach to modeling producer light uptake and productivity. A highly complex, biochemically-based, producerconsumer model of Kuijper, et al [28] also supports a Diehl-like response to both energy and nutrient enrichment. A goal of our study is to clarify the underlying cause of these different enrichment-induced behaviors.

In this paper we present a general stoichiometric producer-consumer model. The model can be viewed as including the essential biological processes of previous models in the literature. The defining equations rely on general but mechanisticallybased submodels of producer and consumer processes and interactions. The basic model is three-dimensional, with dynamic state variables consisting of producer and consumer population sizes, and a measure of producer nutrient content. Although general in nature, the model permits a number of conclusions regarding system dynamics. Through a combination of simulations and numerical bifurcation analysis of a special case parameterized for algae-Daphnia systems, we illustrate the complexity of the dynamics supported by the system. We will demonstrate that the model exhibits the energy enrichment-related bifurcation sequences of Diehl, LKE, and other models, as well as new bifurcation sequences dependent on the total nutrient in the ecosystem. An important contribution of this study is an examination of the sensitivity of our conclusions to alternate approaches to modeling the stoichiometrically-constrained producer primary productivity, as well as the conversion of producer biomass to consumer biomass. This is accomplished by developing a generalized "synthesizing unit" model of biomass production that unifies alternate the approaches used in the LKE, Diehl and other studies.

The general model is presented in Section 2, with a self-contained discussion of the biomass production assumptions given in an appendix. Basic dynamic properties of the general system and the associated monoculture (producer-only) models are examined in Section 3, were it is demonstrated that the results are robust with respect to alternate producer biomass production models. Section 4 reports on a thorough numerical examination of the model in the context of algae-Daphnia 
systems. Model parameterization, summarized in Table 4.1, is based on an extensive review of related models from the literature. In Section 5 we examine the affects of alternate approaches to modeling stoichiometrically-constrained consumer productivity. Model generalizations and limitations are discussed, as well. A summary of our findings and their ecological implications are found in Section 6.

2. Model Derivation. We consider a system consisting of a producer population, $P(t)$, and consumer population, $C(t)$ as described in terms of their spatial carbon densities. Both populations compete for a nutrient, with total amount, $N_{T}$, assumed to be constant. The amount of nutrient sequestered by the consumer is taken to be proportionate to $C(t)$ with $q_{c}>0$ denoting the (assumed) constant nutrient: carbon ratio for the consumer class. See [3] for a discussion of the constancy of consumer stoichiometry. The amount, $N_{P}(t)$, of nutrient sequestered by the producer class is assumed dynamic and consisting of a structural component $q_{p} P(t), q_{p} \geq 0$ and a flexible nutrient reserve, $R(t) P(t)$ needed for the production of new producer biomass. Thus, $R(t)=\left(N_{p}(t) / P(t)\right)-q_{p}$ represents the concentration of nutrients in the flexible nutrient reserves of the producer. Our use of a flexible nutrient reserve is similar in spirit to that of many authors, including [9], [15], [19], [24], and [26].

The model is described by the system

$$
\begin{aligned}
& C^{\prime}=\underbrace{\delta_{c} \mu_{c}\left(l_{c}\right) f(P) P C}_{\text {consumer productivity }}-\underbrace{d_{c} C}_{\text {mortality }} \\
& P^{\prime}=\underbrace{\delta_{p} \mu_{p}\left(l_{p}\right) g(P) P}_{\text {producer productivity }}-\underbrace{d_{p} P}_{\text {mortality }}-\underbrace{f(P) P C}_{\text {predation losses }} \\
& N_{p}^{\prime}=\underbrace{h\left(N_{m}\right) g(P) P}_{\text {nutrient uptake }}-\underbrace{d_{p} N_{p}}_{\text {mortality }}-\underbrace{f(P) N_{p} C}_{\text {predation losses }}
\end{aligned}
$$

where $N_{p}^{\prime}$ is the rate of change of the nutrient pool of the producer.

The terms $l_{p}=\varepsilon_{p} R / \delta_{p} q_{p}$ and $l_{c}=\varepsilon_{c}\left(q_{p}+R\right) / \delta_{c} q_{c}$ define, respectively, producer and consumer growth limitation indices, where $0<\delta_{p}, \varepsilon_{p}, \delta_{c}, \varepsilon_{c} \leq 1$ are assimilation/respiration-related reduction factors described in Appendix A.1. While many of these constants could be absorbed into the definitions of the functions $\mu_{p}$ and $\mu_{c}$, we have elected to retain them for parameterization purposes later.

Because the system is assumed closed, the amount of nutrient not sequestered in producer or consumer biomass (the "mineralized nutrient," $N_{m}$ ) is given by $N_{m}=N_{T}-q_{c} C-N_{p}=N_{T}-q_{c} C-q_{p} P-R P$ and is assumed available for producer uptake. Here, $N_{T}$ denotes the total nutrient pool of the entire system and is a free parameter in our study. Biologically meaningful initial conditions must satisfy $N_{m} \geq 0$. Our goal is to investigate model dynamic behavior, especially the type and sequence of energy enrichment-induced bifurcations as $N_{T}$ varies. 
In order to maximize generality, we do not specify the undefined functions on the right of (2.1-2.2-2.3), but rather assume that they obey certain qualitative properties:

Consumer Productivity. The first term in (2.1) represents the consumer's biomass production rate. The combination $\delta_{c} f(P) P C$ represents the consumer's predation rate reduced by a factor $\delta_{c}<1$ to account for carbon losses due to egestion, digestive processes and respiration. The consumer biomass production rate is further modulated by a conversion efficiency factor $\mu_{c}\left(l_{c}\right)$ that is dependent on the stoichiometric qualities of the consumer's processed food stream in relation to the consumer's stoichiometric needs. The precise form of $\mu_{c}$ is a subject of moderate debate. In Appendix A.1 we show that the standard choices used in the literature can all be derived from a generalized version of the "parallel binding" synthesizing unit construction of Kooijman [25]. Moreover, based on this extended synthesizing unit model of biomass production, we deduce that $\mu_{c}(l)$ is continuous, is non-decreasing and satisfies $l /(1+l) \leq \mu_{c}(l) \leq \min (1, l)$.

Consumer Predation. The specific rate of predation, $f(P) P$ is typified by the classic Holling-II functional response, $f(P) P=\left(f_{\max }^{-1}+(\alpha P)^{-1}\right)^{-1}=f_{\max } P /\left(f_{\frac{1}{2}}+P\right)$, where $f_{\max }^{-1}$ represents the specific handling time, $\alpha$ the effective attack rate, and $f_{\frac{1}{2}}=f_{\max } / \alpha$ defines the predation half-saturation rate. See [34]. In the absence of a handling time, $f_{\max }^{-1} \rightarrow 0$, and one recovers the standard Lotka-Volterra, specific mass-action rate of predation $f(P) P=\alpha P$ as used in [17]. Consistent with [31], we assume that $f(P) P$ is increasing and concave downward in $P$, and that $f_{\max } \equiv \lim _{P \rightarrow \infty} f(P) P<\infty$. Moreover, $f(P)$ is assumed to be non-increasing in $P$. While our numerical experiments will be restricted to the Holling-II form, our analytic results allow inclusion of alternate forms assumed in [2] and [23].

Producer Productivity. For the purpose of studying the impact of light enrichment on model dynamics, we choose to include possible density-dependent reductions in producer growth due to light competition. The factor $g(P)$ in (2.2) represents the maximal (nutrient-unrestricted) specific growth rate for the producer. We assume $g(P)$ to be a non-negative and a decreasing function of $P$ whenever it is positive. Moreover, we assume that for high producer populations, the maximal (nutrient unlimited) specific growth rate is less than its specific natural mortality rate: $\lim _{P \rightarrow \infty} \delta_{p} g(P)<d_{p}$. In the numerical experiments described in Section 4, we take $g(P)=r(b-P / K)^{+}$.

Based on the generalized SU for producer primary production, the factor $\mu_{p}(l)$ is assumed to satisfy the same qualitative properties as $\mu_{c}(l)$. Details are found in Appendix A.2. See [18] for similar qualitative assumptions made on the factor $\mu_{p}(l)$, 
and [45] for an alternate application of the SU to the modeling of producer growth. The case where producer biomass is independent of nutrient can be handled by formally allowing $q_{p} \rightarrow 0$, and setting $\mu_{p}(l) \equiv 1$ in (2.2). If one selects $\mu_{p}(l)=1 /\left(1+l^{-1}\right)$, which in Appendix A.2 is shown to be the lower extreme for the producer biomass reduction factor, one obtains $\mu_{p}\left(l_{p}\right)=1 /\left(1+\frac{\delta_{p} q_{p}}{\varepsilon_{p} R}\right)=R /\left(\frac{\delta_{p} q_{p}}{\varepsilon_{p}}+R\right)$, which is a Michaelis-Menten (Monod) function of producer nutrient reserve density. See, for examples, the models of [6] and [16]. The special case of equal carbon and nutrient efficiencies $\left(\delta_{p}=\varepsilon_{p}\right)$ recovers the Droop [9] biomass factor $\mu_{p}\left(l_{p}\right)=1 /\left(1+q_{p} / R\right)=1-q_{p} /\left(R+q_{p}\right)$ often selected in the literature ([2], [7], [8], [17], [20], [26], [35], among others.)

Producer Nutrient Dynamics. Producer mortality (whether due to predation or not) induces a proportionate reduction in producer nutrient pool, $N_{p}$. We assume that any producer nutrient lost due to natural death, or lost due to predation but in excess of the biomass production needs of the consumer, immediately joins the pool of mineralized nutrient, $N_{m}=N_{T}-q_{c} C-\left(R+q_{P}\right) P$. The nutrient uptake rate is assumed proportionate to the producer maximal specific growth rate, with proportionality constant $h=h\left(N_{m}\right)$ a smooth, non-negative, increasing function of non-sequestered nutrient. Additionally, we make the mild technical assumption that $h(n) \leq h^{\prime}(0) n \equiv \beta n$ for some $\beta>0$. For the purposes of numerical simulation we will use the Michaelis-Menten form $h(n)=\left(h_{\max }^{-1}+(\beta n)^{-1}\right)^{-1}$, and explicitly allow the unsaturated case $h(n)=\beta n$ corresponding to $h_{\max }^{-1} \rightarrow 0$.

The assumed dependence of nutrient uptake on producer productivity is common to the models of [22] and [4]. By mass-balance arguments this dependence is required of models where the producer stoichiometry is fixed, as in [18] and [6]. However, a common, alternate approach is to assume that producer nutrient uptake is proportionate to $P$, with proportionality constant taken to be both a saturating function of mineralized nutrient density, $N_{m}$, and a decreasing function of producer internal stores, $R$. See, for examples, [2], [7] and [48]. In contrast, the models of [36], [20], [47] and [49] assume that nutrient uptake is proportionate to $P$, with a proportionality constant that is a saturating function of $N_{m}$, but independent of internal stores. Our assumption provides certain technical advantages, as will be seen in Section 4 and in the mathematical analysis of $[43,44]$. Moreover, Diehl [8 (Appendix A)] cites evidence that the details of nutrient uptake may not be a significant influence on system dynamics.

It is helpful to the analysis of this system to replace (2.3) with an equivalent differential equation for producer nutrient reserve concentration, $R$. Differentiating the relation $R+q_{p}=N_{p} / P$ and using (2.2) and (2.3) results in 


$$
R^{\prime}=g(P)\left[h\left(N_{T}-q_{c} C-q_{p} P-R P\right)-\delta_{p} \mu_{p}\left(\frac{\varepsilon_{p}}{\delta_{p}} \frac{R}{q_{p}}\right)\left(R+q_{p}\right)\right]
$$

3. Model Properties In Appendix B.1 we show that regardless of the selection of constituent functions and model parameters, the general model is well-posed in the sense that solutions with non-negative initial conditions remain non-negative and bounded for all time. Specifically, Proposition B.1 implies that when $q_{p}>0$ solutions of $(2.1,2.2,2.3)$ are uniformly bounded within the "biologically feasible" region $0 \leq q_{c} C+q_{p} P \leq N_{T}$. For the case $q_{p}=0$, uniform boundedness can be shown using elementary methods.

While our primary goal is to understand the effects of enrichment on the dynamics of $(2.1,2.2,2.3)$, we first show that under low energy and/or nutrient levels the system cannot sustain a producer population. It immediately follows from (2.1) that the consumer population cannot be sustained, as well.

Theorem 3.1 If either $\delta_{p} g(0)<d_{p}$ or $h\left(N_{T}\right) g(0)<d_{p} q_{p}$, then $P(t) \rightarrow 0$ as $t \rightarrow \infty$.

Proof: Under the first inequality the conclusion follows from (2.2) using the properties that $\mu_{p} \leq 1$ and that $g(0) \geq g(P)$. For the second case we use (2.3) and the monotonicity of $h$ to find $\left.N_{p}^{\prime} \leq h\left(N_{m}\right) g(0) P-d_{p}\left(R+q_{p}\right) P \leq\left[h\left(N_{T}\right) g(0)-d_{p} q_{p}\right)\right] P$. From $N_{p}=\left(R+q_{p}\right) P \geq q_{p} P$ it follows that $N_{p}(t) \rightarrow 0$ as $t \rightarrow \infty$, and therefore $P(t) \rightarrow 0$.

In this theorem, the first case can be interpreted to mean that producer persistence requires sufficiently high biomass production relative to its specific mortality rate. The second case states that if the producer is nutrient-limited, then producer persistence requires that the nutrient uptake must potentially be larger than the producer's loss of structural nutrient due to natural mortality. Since $h\left(N_{T}\right) \rightarrow 0$ as $N_{T} \rightarrow 0$, this can also be interpreted to say that system persistence requires a sufficiently large total system nutrient pool. The following theorem complements the previous, showing that for sufficiently high energy levels, there is a positive monoculture equilibrium that (in the absence of consumer) is globally attracting.

Theorem 3.2 If $q_{p}=0\left(\mu_{p} \equiv 1\right)$, and $\delta_{p} g(0)>d_{p}$ then there is a unique monoculture equilibrium $\left(0, \bar{P}^{m}, \bar{R}^{m}\right)$ to system $(2.1,2.2,2.4)$ and this equilibrium is globally attracting to all solutions with $C(0)=0$. If $q_{p}>0$, then the same conclusion holds if $\delta_{p} \mu_{p}\left(\frac{\varepsilon_{p}}{\delta_{p}} \frac{R_{\max }}{q_{p}}\right) g(0)>d_{p}$ where $R_{\max }$ is implicitly defined as the unique solution of the equation $\delta_{p} \mu_{p}\left(\frac{\varepsilon_{p} R_{\max }}{\delta_{p} q_{p}}\right)\left(R_{\max }+q_{p}\right)=h\left(N_{T}\right)$.

The proof, which relies on a nullcline analysis for the reduced system $(2.2,2.4)$ with $C \equiv 0$, is found in Appendix B.2. A transcritical bifurcation for the producer class 
corresponds to the situation when system nullclines intersect at $P=0$. Such bifurcations can be computed in terms of the producer nutrient content, as illustrated in Figure 3.1. Specifically, the figure shows the producer transcritical bifurcation curves based on the Liebig, Poisson Arrival Time (PAT) and Michaelis-Menten (Monod) cases. The horizontal axis, the maximal, nutrient-unlimited specific producer primary production rate, can be a viewed as a measure of energy enrichment of the system. The vertical axis describes the nutrient level of the system as perceived by the producer's maximum possible level of nutrient uptake, $h_{T} \equiv h\left(N_{T}\right)$. The producer transcritical bifurcation curves are seen to be qualitatively similar, regardless of the selection of $\mu_{p}$. Note that for a given level of energy enrichment $r \equiv g(0)$, as the biomass conversion factor $\mu_{p}$ decreases from the Liebig to the Michaelis-Menten (Monod) case (see Figure A.1), persistence of the producer monoculture system requires higher producer nutrient uptake, $h_{T}$.

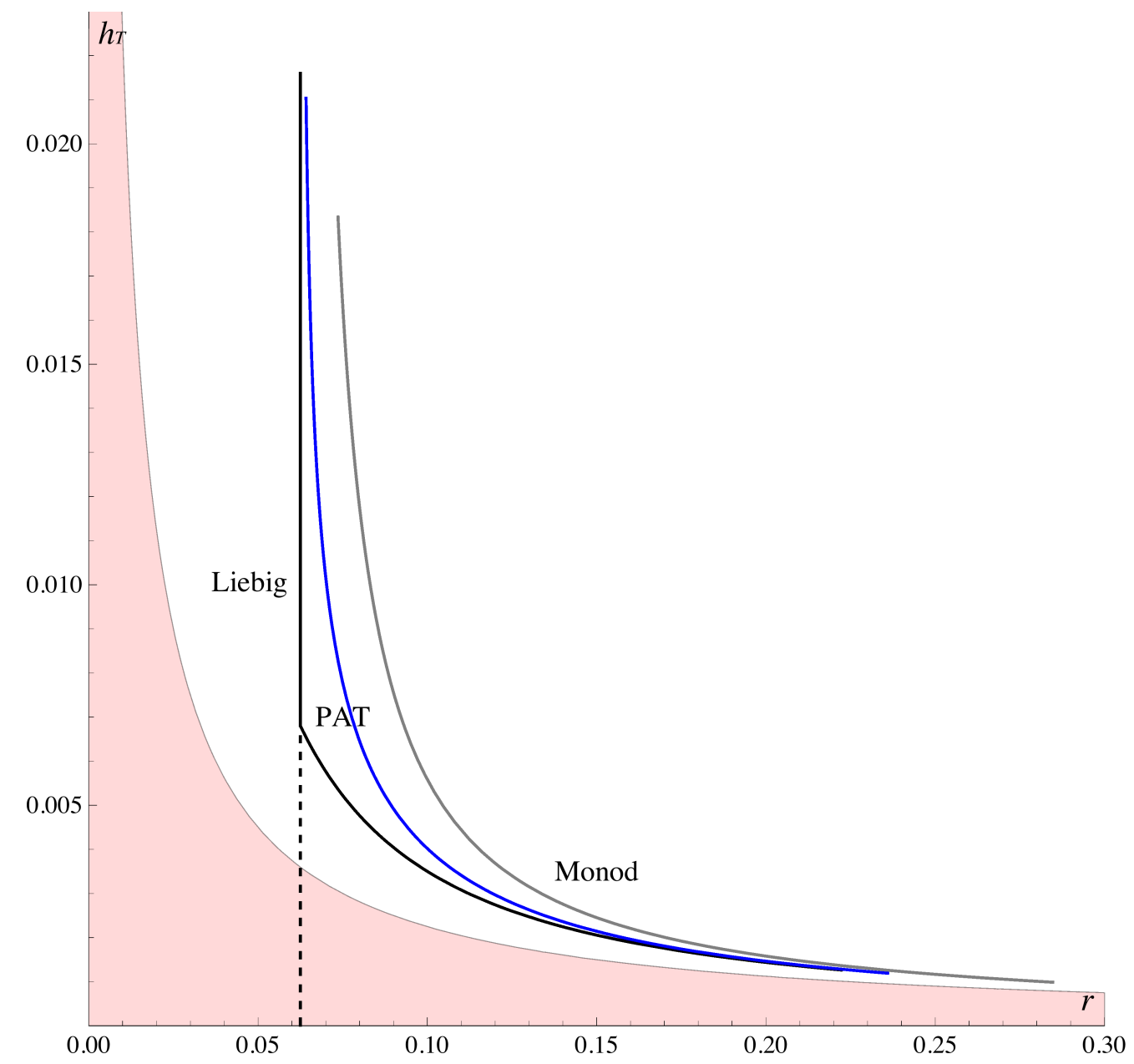

Figure 3.1: Curves bounding the transcritical bifurcation to a globally attracting monoculture steady-state, along with the transcritical curve (blue) for the Poisson Arrival Time (PAT) case $\mu_{p}(l)=\left(1+l^{-1}-(1+l)^{-1}\right)^{-1}$. Transcritical curves are computed parametrically in terms of the producer nutrient reserve concentration, $R$, 
from $r=g(0)=d_{p} / \delta_{p} \mu_{p}\left(\frac{\varepsilon_{p}}{\delta_{p}} \frac{R}{q_{p}}\right)$ and $h_{T}=h\left(N_{T}\right)=\delta_{p} \mu_{p}\left(\frac{\varepsilon_{p} R}{\delta_{p} q_{p}}\right)\left(R+q_{p}\right)$ Left and right bounds are generated using Liebig upper bound $\mu_{p}(l)=\min [l, 1]$ and MichaelisMenten (Monod) lower bound $\mu_{p}(l)=\left(1+l^{-1}\right)^{-1}$, respectively. In the case $q_{p}=0$, $\mu_{p} \equiv 1$ the transcritical curve reduces to the vertical line $r=d_{p} / \delta_{p}$. The two conditions for global stability of the "no-life" equilibrium (Theorem 3.1) are represented, respectively, by the region to the left of the vertical line, and by the shaded region. In all cases $l=\frac{\varepsilon_{p}}{\delta_{p}} \frac{R}{q_{p}}$ with parameters $d_{p}=.05, \delta_{p}=.8, \varepsilon_{p}=.9$, $q_{p}=.0045$, and $.25 q_{p} \leq R \leq 5 q_{p}$. See Section 4 for parameter selections and units.

In contrast to the general results of this section, here we next present numerical tests for a specific realization of $(2.1,2.2,2.4)$. Using simple differential inequalities it is easy to show that the consumer population cannot persist if its maximal (nutrient unlimited) biomass production rate is less than its mortality rate. Thus, we will proceed under the assumption that that $\lim _{P \rightarrow \infty} \delta_{c} f(P) P>d_{c}$.

\section{Numerical Studies for Algae-Daphnia Systems}

In this section we demonstrate that for biologically reasonable selections of model functions parameters, the model $(2.1,2.2,2.4)$ supports a wide variety of enrichmentinduced changes in system dynamics, including the distinct bifurcation sequences for the models of [8] and [31]. We observe new bifurcation sequences, as well. Function and parameter selections are motivated by pelagic studies. For such systems one normally considers phosphorous as the limiting nutrient [11]. In this context, $P$ and $C$ will be described in terms of mg carbon per liter, while $R$ is in terms of $\mathrm{mg}$ phosphorous/mg carbon.

While nutrient enrichment corresponds to a simple increase in total system nutrient, $N_{T}$, light enrichment is described in terms of its effect on $g(P)$. Our numerical experiments employ the simple empirical choice $g(P)=\max [r(b-P / K), 0]$. Here $r b>0$ defines the maximal intrinsic rate of producer growth, $b$ denotes the enrichment parameter ( $b=1$ corresponding to the default level), and $K$ plays a role similar to the producer carrying capacity.

Our selection of $g(P)$ is motivated by that of [6], [7], [8], and [48], who apply the spatially-aggregated light gradient model of Huisman \& Weissing ([21], [22], [50]) to aquatic systems. With a slight variation in notation, these authors take $g(P)=\frac{1}{z} \int_{0}^{z} \psi\left(I_{0} e^{-\left(\kappa P+\kappa_{b}\right) s}\right) d s$, where $z$ denotes the depth of the water column, $\psi(I)$, defines the rate of producer productivity at light level $I$, and (based on the BeerLambert Law) $I=I_{0} e^{-\left(\kappa P+\kappa_{b}\right) s}$ models the light level at depth $s, 0 \leq s \leq z$. The parameters $\kappa$ and $\kappa_{b}$ account for light absorption by the producer and ambient 
"background" particulate matter, respectively, while $I_{0}$ denotes the light intensity at water surface level. Light enrichment can be accomplished by increasing $I_{0}$, or decreasing $z$ or $\kappa_{b}$. In the aforementioned references, the function $\psi$ is taken of Michaelis-Menten type, but more generally can be simply assumed increasing in $I$, with $\psi(0)=0$.

Changing variables in the integral, one has $g(P)=\int_{0}^{1} \psi\left(I_{0} e^{-\left(\kappa P+\kappa_{b}\right) z u}\right) d u$. By simple differentiation one can see that for $P \geq 0, g(P)$ is decreasing in $P$. Moreover, for each fixed $P \geq 0, g(P)$ is strictly increasing under enrichment, regardless of the specific enrichment mechanism. In fact, an increase in the nutrient-saturated $\quad\left(\mu_{p}=1\right)$ producer growth rate provides an operational definition of energy enrichment in our investigation.

Additionally, we select $\mu_{p}(l)=\left(l^{-1}+1\right)^{-1}=l /(1+l)$ (Michaelis-Menten producer biomass conversion), $f(P) P=\left(f_{\max }^{-1}+(\alpha P)^{-1}\right)^{-1}=f_{\max } P /\left(f_{\frac{1}{2}}+P\right)$ (Holling II predator functional response) $h(n)=\left(h_{\max }^{-1}+(\beta n)^{-1}\right)^{-1}=h_{\max } n /\left(h_{\frac{1}{2}}+n\right)$ (Michaelis-Menten -based producer nutrient uptake) and $\mu_{c}(l)=\left(l^{-1}+1-(1+l)^{-1}\right)^{-1}$ (Poisson Arrival Time type consumer biomass synthesizing unit). Enrichment results in a proportionate increase in maximal specific producer growth rate, $g(0)=r b$, as well as an increase in producer monoculture carrying capacity, $\bar{P}^{m}$.

Table 4.1 gives the values for each of the 16 model parameters used in our model simulations. The work of Andersen [2] provides the source of many of these selections. In particular, we refer to that reference's thorough discussion of algal maximum specific growth rate $r$, structural nutrient concentration $q_{p}$ (there called the "subsistence quota") and nutrient uptake affinity, from which our parameter $\beta$ is estimated. In many cases the parameter values from cited the references require conversion to the units used here

Table 4.1: Model Parameters.

\begin{tabular}{|l|l|l|l|}
\hline Parameter & Units & Default and Range & References \\
\hline $\begin{array}{l}\text { Maximal producer specific } \\
\text { growth rate, } r\end{array}$ & day $^{-1}$ & $1.1,(1.0,3.0)$ & {$[31],[47],[35],[48],[8],[3]$} \\
\hline $\begin{array}{l}\text { Maximal producer carrying } \\
\text { capacity, } K\end{array}$ & $\mathrm{mg} P$ carbon/l & $1.25,(.25,3.0)$ & {$[31],[45]$} \\
\hline $\begin{array}{l}\text { Producer specific natural } \\
\text { mortality rate }(\text { no } \\
\text { respiration), } d_{p}\end{array}$ & day $^{-1}$ & $.05,(.01, .1)$ & {$[31],[45],[35],[48],[8],[3]$} \\
\hline
\end{tabular}




\begin{tabular}{|c|c|c|c|}
\hline $\begin{array}{l}\text { Consumer specific mortality } \\
\text { rate (no respiration), } d_{c}\end{array}$ & day $^{-1}$ & $.24,(.02, .3)$ & [31], [47], [35], [48], [8], [3] \\
\hline $\begin{array}{l}\text { Maximal producer carbon } \\
\text { assimilation/ respiration } \\
\text { efficiency, } \delta_{p}\end{array}$ & unitless & $.8,(.5,1.0)$ & [17] \\
\hline $\begin{array}{l}\text { Maximal producer nutrient } \\
\text { assimilation efficiency, } \varepsilon_{p}\end{array}$ & unitless & $.9,(.5,1.0)$ & [17] \\
\hline $\begin{array}{l}\text { Maximal consumer carbon } \\
\text { assimilation /respiration } \\
\text { efficiency, } \delta_{c}\end{array}$ & unitless & $.8,(.5,1.0)$ & {$[16],[17],[20],[5]$} \\
\hline $\begin{array}{l}\text { Maximal consumer nutrient } \\
\text { assimilation efficiency, } \varepsilon_{c}\end{array}$ & unitless & $.9,(.5,1.0)$ & [16], [17], [20], [5] \\
\hline $\begin{array}{l}\text { Maximal consumer specific } \\
\text { predation rate, } f_{\text {max }}\end{array}$ & day $^{-1}$ & 1., $(.75,1.0)$ & [31], [47], [35], [48], [8], [3] \\
\hline $\begin{array}{l}\text { Consumer predation half- } \\
\text { saturation constant, } f_{\frac{1}{2}}\end{array}$ & $\operatorname{mg} P$ carbon $/ \mathrm{l}$ & $.2,(.12, .25)$ & {$[31],[47],[35],[48],[8],[3]$} \\
\hline $\begin{array}{l}\text { Producer minimal } \\
\text { ("structural") nutrient: carbon } \\
\text { ratio, } q_{p}\end{array}$ & $\begin{array}{l}\text { mg nutrient /mg } \\
\text { carbon }\end{array}$ & $.0045,[0, .0085)$ & {$[31],[47],[35],[48],[8],[3]$} \\
\hline $\begin{array}{l}\text { Consumer nutrient: carbon } \\
\text { ratio, } q_{c}\end{array}$ & $\begin{array}{l}\text { mg nutrient /mg } \\
\text { carbon }\end{array}$ & $.0375,(0.01, .04)$ & [31], [47], [35], [48], [8], [3] \\
\hline $\begin{array}{l}\text { Producer growth-specific } \\
\text { nutrient uptake affinity, } \beta\end{array}$ & $\mathrm{l} / \mathrm{mg}$ nutrient & $6.0,(0.1,25)$. & [46], [48], [2] \\
\hline $\begin{array}{l}\text { Producer maximal growth- } \\
\text { specific nutrient uptake, } h_{\max }\end{array}$ & $\begin{array}{l}\text { mg nutrient } / \mathrm{mg} \\
\text { carbon }\end{array}$ & $.1,(0.05,1)$. & [47] \\
\hline Total system nutrient, $N_{T}$ & mg nutrient/l & $.02,(0.0031, .031)$ & {$[31],[47],[35],[48],[8],[3]$} \\
\hline Energy enrichment factor, $b$ & Unitless & $1,(0,2)$ & - \\
\hline
\end{tabular}

Numerical simulations of systems $(2.1,2.2,2.4)$ were performed with Mathematica 7.0 [33], while numerical bifurcation computations were performed with AUTO [10]. AUTO computations confirmed simulation observations, and provided a full picture of enrichment-induced bifurcations (total system nutrient, $N_{T}$, fixed), as well as a twoparameter bifurcation summary where enrichment level, $b$, and total system nutrient varied simultaneously. 
Figure 4.1 shows the two-dimensional bifurcation structure for the system $(2.1,2.2$, $2.4)$ using the default parameter values of Table 4.1. The energy enrichment factor varies over $0 \leq b \leq 2$ and total system nutrient level ranges over $0 \leq N_{T} \leq .03$.

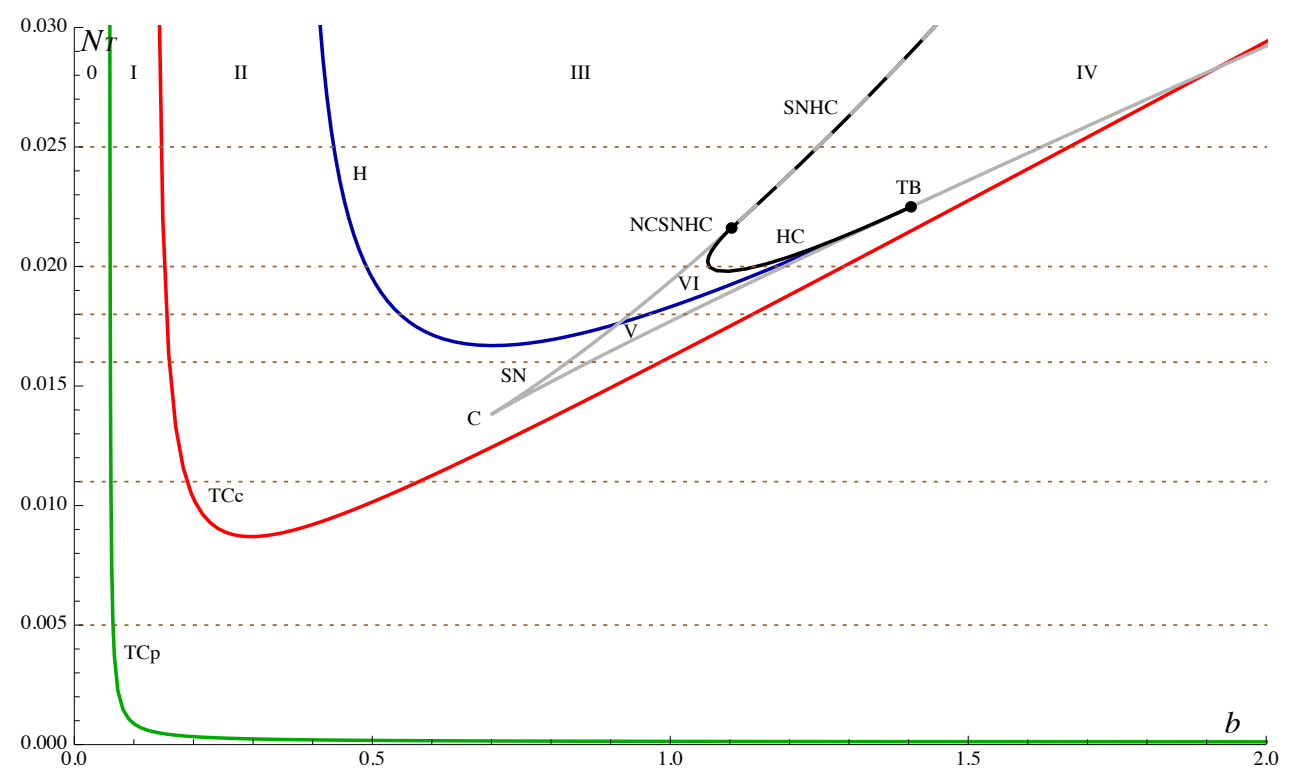

Figure 4.1: The two-parameter bifurcation diagram for model $(2.1,2.2,2.4)$, using default parameters of Table 4.1. Shown are the transcritical bifurcation curves associated with the creation of monoculture equilibria $\left(T C_{p}\right)$ and coexistence equilibria $\left(T C_{c}\right)$. Hopf bifurcations from coexistence equilibria are located along blue curve $H$. Saddle-node creation/loss of coexistence equilibria are on grey curves $S N$, with standard cusp point $C$. With increasing enrichment, $b$, periodic solutions can be lost as homoclinic bifurcations (along black curve $H C$ ) or as homoclinic bifurcations simultaneous to saddle node bifurcations. These latter type bifurcations (dashed curve $S N H C$ ), are referred to as "saddle-node Homoclinic" bifurcations [10]. Numerical computations indicate a Takens-Bogdanov point located at $T B$, which serves as a terminal point of both the Hopf bifurcation and homoclinic bifurcation curves. A "noncentral saddle-node homoclinic" bifurcation point [29], denoted by $N C S N H C$, locates the joining of the curve of homoclinics and the saddle node curve. See [44] and [45] for similar diagrams computed for specific two- dimensional, producer-consumer models derived under different producer growth and nutrient uptake assumptions. Dashed horizontal lines locate nutrient levels associated with six specific energy enrichment scenarios described in the text.

To elucidate Figure 4.1, six one-parameter bifurcation diagrams for selected fixed nutrient levels, $N_{T}$ are shown in Figure 4.2. These diagrams demonstrate the effects of total system nutrient levels on energy-enrichment induced changes in system dynamics. Observe that for each fixed nutrient level, high energy enrichment always 
results in the ultimate re-stabilization of the producer monoculture equilibrium, similar to the behavior of the LKE [31] model.

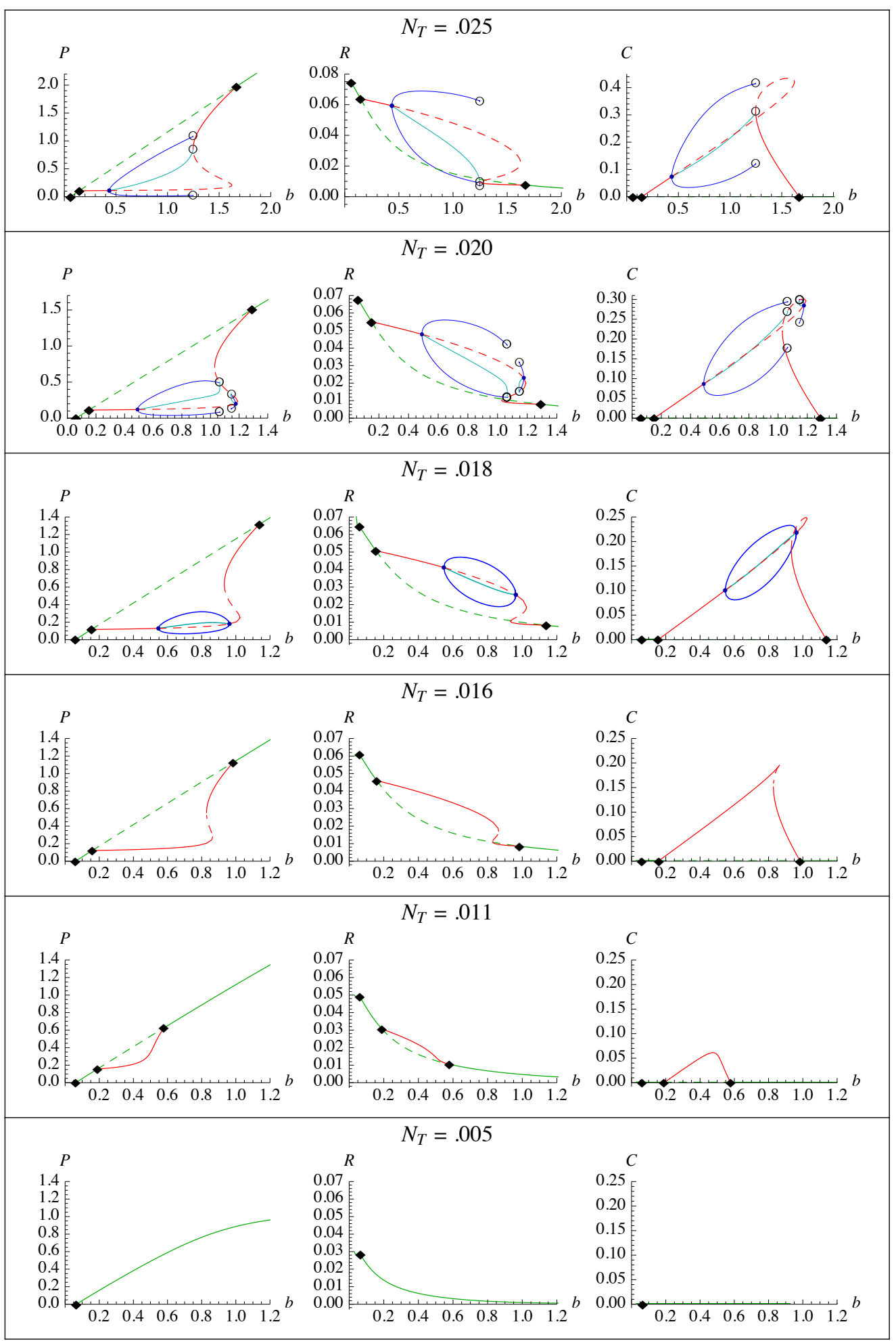

Figure 4.2: One-parameter bifurcation diagrams based on default parameter values from Table 4.1. Solid lines denote stable equilibria/periodic orbits; dashed curves are 
unstable. For enrichment intervals where the model supports periodic orbits, solution maxima, and minima (blue) and average values (cyan) are plotted. Black diamonds locate transcritical bifurcation points for equilibria, while filled circles locate points of Hopf and open circles denote homoclinic bifurcations. For the sake of clarity, saddle-node bifurcations (turning points on the red curves) are not labeled. (See Figure 4.3, below, for full annotation.) The computed maximum concentrations for the producer flexible nutrient pool (center column) are consistent with the phosphorous storage capacity values cited in Andersen [2].

For low nutrient levels $\left(N_{T}=.005\right)$ energy enrichment results in the creation of a producer monoculture equilibrium, which by Section 3 is globally attracting. With further energy enrichment, the increase in monoculture equilibrium causes (by dilution) a reduction in the nutrient concentration of the producer flexible nutrient pool.

At $N_{T}=.011$ there is an intermediate interval of energy enrichment for which the system supports a non- trivial coexistence equilibrium. Numerical simulations indicate that the coexistence equilibrium is globally stable. Under high energy enrichment, the co-existence equilibrium is lost in a second transcritical bifurcation, signaling the return of a globally stable producer monoculture equilibrium.

For $N_{T}=.016$, increasing energy enrichment causes a transcritical creation of a locally stable, high food quality (large $R$ ) coexistence equilibrium. Further enrichment induces a saddle-node creation of a pair of coexistence equilibria with larger producer levels and lower food quality. The larger of the pair (as measured in terms of producer size) is locally attracting, while the smaller is an unstable saddle. With further increase in energy enrichment the unstable saddle combines with the high food-quality coexistence equilibrium and both are lost in a saddle-node bifurcation. Subsequent to this bifurcation, the sole remaining coexistence equilibrium is locally attracting, but (with increasing enrichment) is lost in a transcritical bifurcation that leaves only a producer monoculture equilibrium, which globally attracting.

Increasing total system nutrient to $N_{T}=.018$ complicates the previous case in that energy enrichment destabilizes the high-food quality equilibrium to a (supercritical) stable Hopf bifurcation. For the periodic state, mean food quality is observed to be slightly less than that of the (unstable) high-food quality equilibrium, both of which decline under increased enrichment. With further energy enrichment, this periodic orbit is lost in a Hopf bifurcation, signaling the re-stabilizing of the high food-quality equilibrium state. As indicated by Figure 4.1, through variation of total system nutrient, this loss of periodic coexistence can occur either after (as pictured) or before a saddle-node creation of low food quality coexistence equilibria.

Qualitatively, this loss of periodic state is the same as that observed in the 2D producer-consumer model of Diehl [8]. 
For $N_{T}=.020$, the $b$ vs. $P$ one-parameter diagram from Figure 4.2 is annotated and enlarged in Figure 4.3 below. We again observe an enrichment-induced Hopf bifurcation from the high food quality coexistence equilibrium. This periodic state is lost in a homoclinic bifurcation near $b=1.07$. Further energy enrichment induces the creation of a stable periodic solution via a subsequent homoclinic bifurcation. This periodic solution is lost in a Hopf bifurcation (re-stabilization of the high food quality coexistence equilibrium.) With a small additional increase in energy enrichment this equilibrium state is lost in a saddle-node bifurcation. Observe that for the range $.9 \leq b \leq 1.3$ (corresponding to a $10 \%$ energy reduction to a $30 \%$ increase) the number of stable coexistence states varies abruptly.

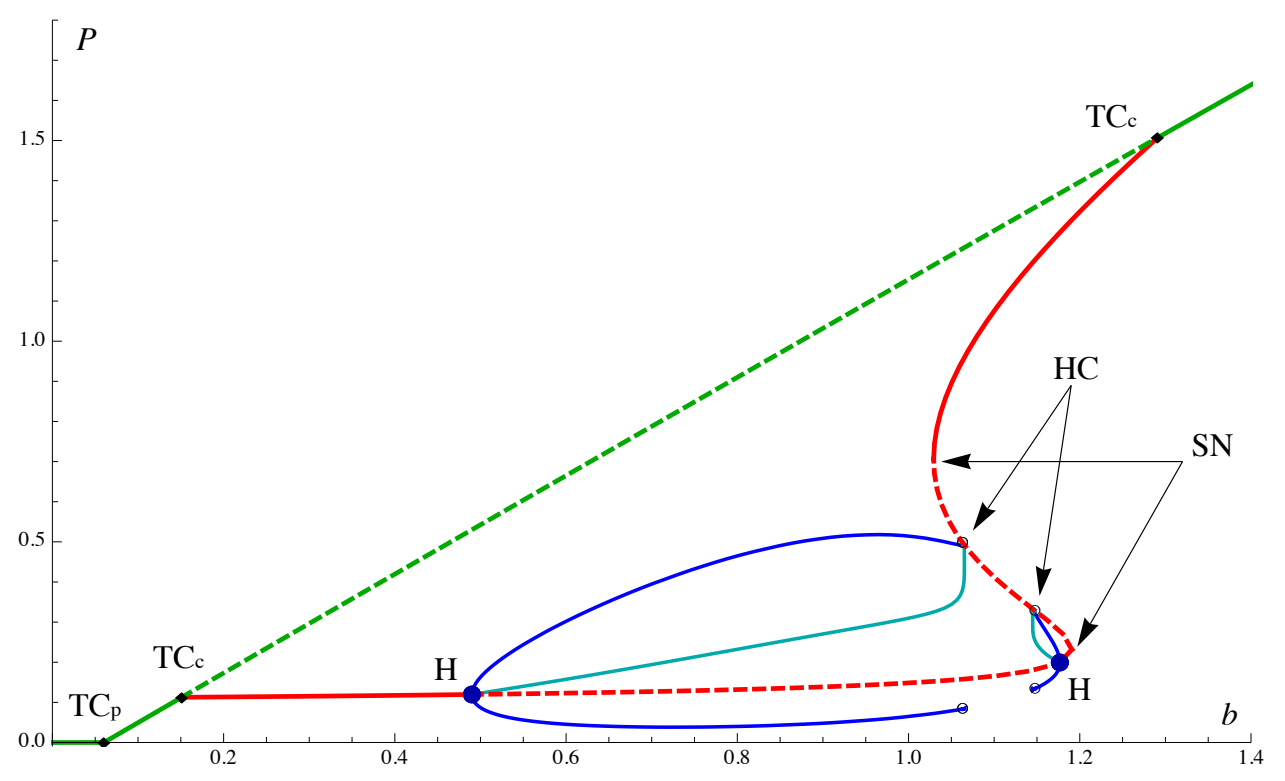

Figure 4.3: Annotated view of the one-parameter bifurcation diagram for $N_{T}=.020$ (producer coordinate). Bifurcation points are labeled as in Figure 4.1. The stable periodic solution is lost in a homoclinic bifurcation near $b=1.07$. However, with further energy enrichment there is a subsequent homoclinic creation of stable periodic solution near $b=1.15$. This periodic family then terminates in a Hopf bifurcation just prior to the saddle-node loss of the high food quality equilibrium near $b=1.19$.

To better understand the interpretation of this figure, it is useful to consider solution trajectories in relation to the nullclines associated with a two-dimensional "quasiequilibrium" system. For this, (2.1) and (2.2) are coupled with the algebraic equation

$$
h\left(N_{T}-q_{p} P-R P\right)=\delta_{p} \mu_{p}\left(\varepsilon_{p} R / \delta_{p} q_{p}\right)\left(R+q_{p}\right)
$$


obtained under the assumption that $R^{\prime} \equiv 0$. For our selections for $h$ and $\mu_{p}$ the solution of (4.1) can be computed as the root of a cubic polynomial, solvable with the technical assistance of Mathematica. Figure 4.4 shows "hybrid" phase plots for the case $N_{T}=.02$, in which the $P C$ projections of periodic solutions to $(2.1,2.2,2.4)$ are viewed along with the computed flow lines and nullclines for the quasi-equilibrium system $(2.1,2.2,4.1)$.
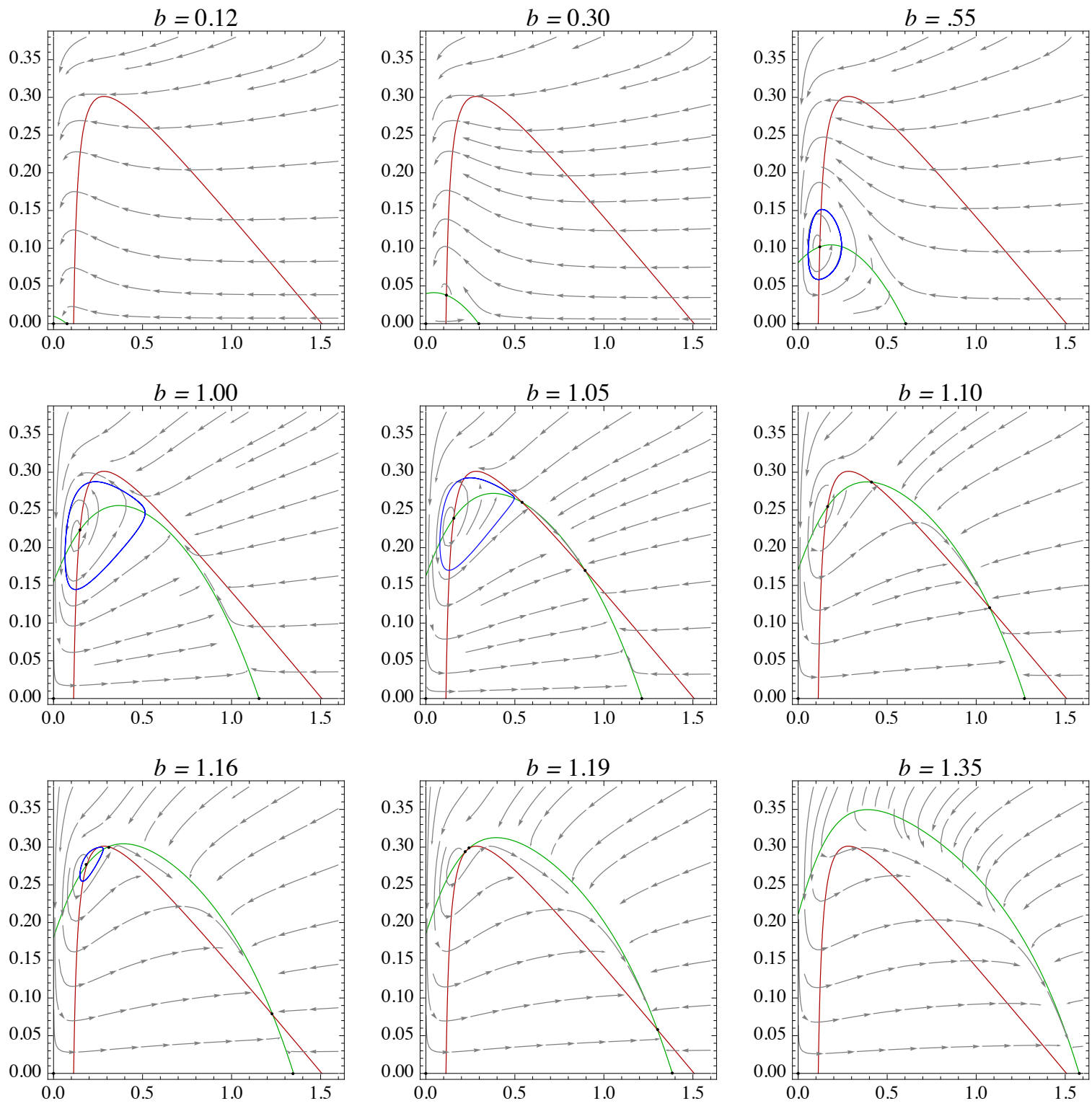

Figure 4.4: Representative quasi-equilibrium "hybrid" phase plane diagrams for $N_{T}=.02$. All horizontal axes $=P$; vertical axes $=C$. Red denotes the consumer nullcline; green denotes the producer nullcline, both computed under the "quasiequilibrium" assumption that $R^{\prime} \equiv 0$. Blue curves are $P C$ projections of the periodic orbits for the full system $(2.1,2.2,2.4)$. Other pictured solution flow lines are based on the quasi-equilibrium approximation. The initial Hopf bifurcation is seen to occur 
at a value of $b$ slightly less than .5. A saddle-node creation of a pair of low food quality coexistence equilibria occurs for $b$ near 1.03, prior to the homoclinic loss of the attracting periodic solution near $b=1.07$. Further energy enrichment induces the homoclinic re-creation of an attracting periodic state near $b=1.15$. This periodic state terminates in a Hopf bifurcation near $b=1.18$. For the enrichment interval between the two homoclinic bifurcations, the coexistence equilibrium with lowest food quality is essentially attracting of all interior solutions. The pair of high food quality equilibria is lost via a saddle-node bifurcation near $b=1.20$, and the final transcritical bifurcation occurs near $b=1.29$.

For high system nutrient (here represented by case $N_{T}=.025$ ), the general oneparameter bifurcation structure is similar to that reported for the LKE model [31]. This case differs from $N_{T}=.020$ in that the stable periodic orbit created via the destabilization of the high food quality coexistence equilibrium is lost in a homoclinic periodic orbit simultaneous to the saddle-node creation of low food quality coexistence equilibrium $(S N H C)$. A representative "hybrid" phase diagram for enrichment values slightly lower than this homoclinic/saddlenode bifurcation is shown in Figure 4.5.

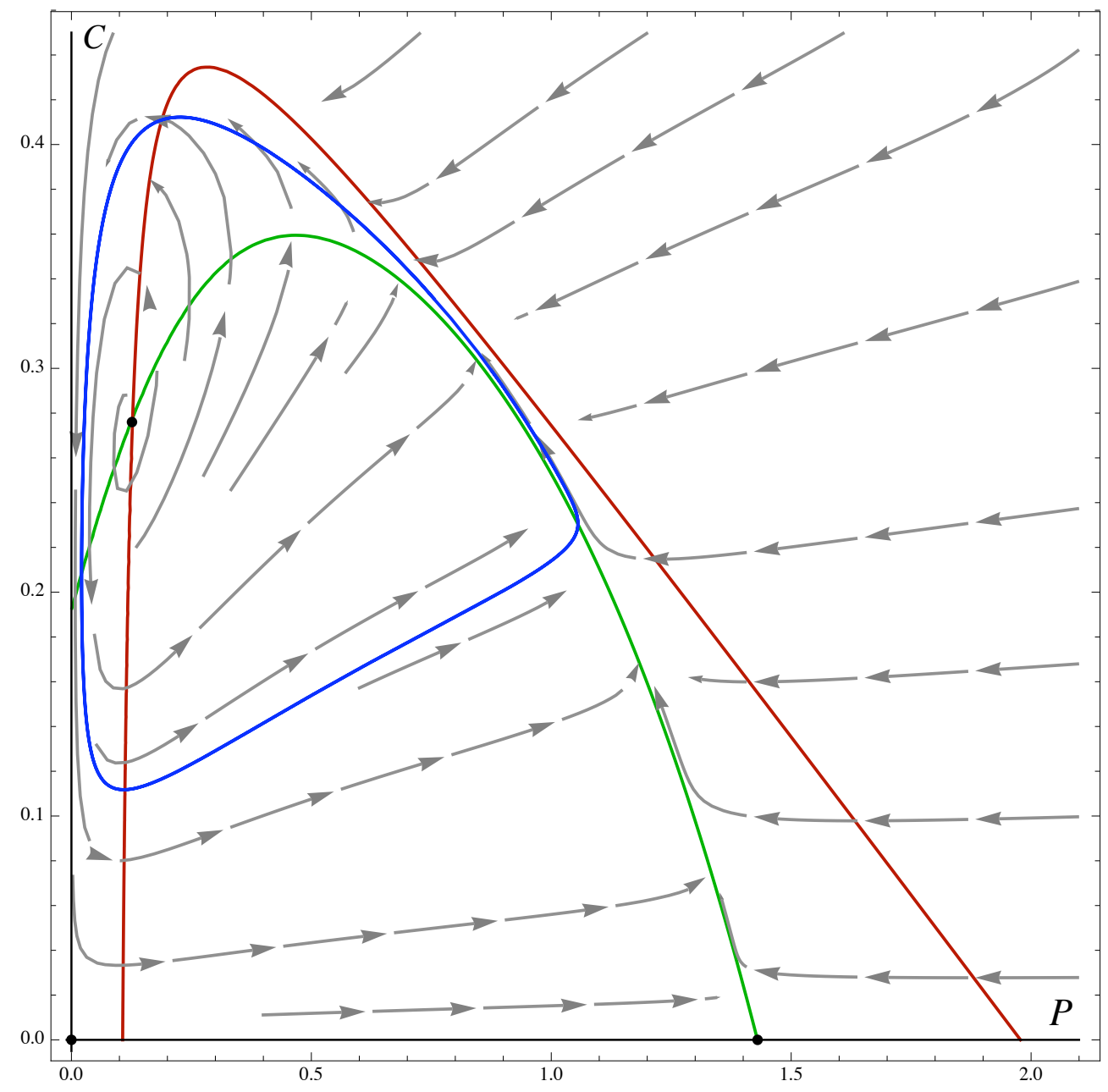


Figure 4.5: "Hybrid" Phase plane diagrams for $N_{T}=.025$ and $b=1.22$. With increased enrichment, the periodic orbit (shown in blue) is lost in a homoclinic bifurcation simultaneous to the saddle-node creation of two low-food quality equilibria born when the (green) producer nullcline intersects the (red) consumer nullcline at approximately $(P, C)=(.8, .3)$. The larger of the two low food quality coexistence equilibria becomes attracting of essentially all positive solution trajectories, as depicted in Figure 4.4 with $b=1.10$. The discrepancy between the periodic orbit and the flow lines is due to the "quasi-equilibrium" approximation used in computing the latter. However, there seems to be a general consistency between the two systems. A rigorous comparison of system $(2.1,2.2,2.4)$ and this twodimensional approximation is given in [44].

In summary, based on these calculations it appears that the bifurcation curves shown in Figure 4.1 partition the $\left(b, N_{T}\right)$ plane into distinct regions of system dynamics. In region 0 energy/nutrient levels are insufficient to sustain the producer monoculture. In region I the producer monoculture exists, while in region II the producer and consumer can coexist in a stable equilibrium. In region III there is an unique coexistence equilibrium (unstable) and an attracting periodic coexistence state. In region IV effectively all solutions approach a low food quality coexistence equilibrium. Regions V and VI are regions of bistability, with the system supporting a locally attracting low food quality equilibrium state simultaneous to a locally attracting high food quality equilibrium (V) or attracting periodic coexistence state (VI). It is not known to what extent to this partition of the $\left(b, N_{T}\right)$ plane is affected by changes in the constituent functions and other auxiliary parameter selections.

\section{Discussion}

The model considered here demonstrates the effects that variable producer stoichiometry can have on models of nutrient-constrained, energy enriched systems. Our findings are summarized in Figure 4.3. Beyond a minimal critical system nutrient level, energy enrichment induces the creation of a monoculture equilibrium state consisting of the producer only. Under higher nutrient levels, energy enrichment can cause this state to lose stability, signaling that the system can support a coexistent consumer population and a locally-attracting coexistence equilibrium. With sufficient additional nutrient, the system can support a periodic coexistence state, but only for an intermediate level of energy enrichment levels. Under very high nutrient levels energy enrichment induces a loss of periodic solutions in an infinite-period (homoclinic) bifurcation. The numerical experiments of Section 4 illustrate the diversity of dynamic changes that can occur at intermediate energy levels. A number of possible scenarios is observed, including the homoclinic creation of attracting highperiod coexistence solutions and/or the creation of new attracting coexistence equilibria. 
Our results unify the results of LKE [31], [27] and Diehl [8], in that under energy enrichment periodic coexistence here is lost through a variety of bifurcation sequences dependent on the total system nutrient level (Figure 4.2). At lowintermediate nutrient levels the model resembles the Diehl model, while at high nutrient levels it resembles the LKE model. Moreover, at intermediate nutrient levels, we observe more complicated bifurcation sequences that simultaneously share the characteristics of both the LKE and Diehl models. As is the case for those models, under high energy levels the system supports a globally attracting equilibrium state. The results presented here provide useful motivation for a rigorous analysis of the quasi-equilibrium approximation $(2.1,2.2,4.1)$ and an examination of its similarities and differences to the general model of this paper [44].

Regarding the Diehl study, we note that the impact of altering enrichment by way of varying water column depth is strictly speaking not covered by our analysis. In the Diehl model, producer and consumer populations are assumed homogeneously spatially distributed and (as pointed out in Section 4), the model of producer primary productivity used there satisfies the assumptions of our model. However, the Diehl model is not closed in nutrient, with algae (but not its consumer) allowed to leave the system due to sedimentation. Therefore, changing water column depth not only affects energy levels, but also influences algae sinking losses from the system -- hence the nutrient pool. However, the general characteristics of the producer and consumer nullclines shown for the simplified model in [8], as well as their dependence on system nutrient levels and decreased water column depth, are consistent with our results.

\section{Model Robustness: Altering the Consumer Biomass Conversion Submodel}

As a multi-parameter class of models with qualitatively defined constitutive functions, it is difficult to draw general conclusions about the extent that the observed enrichment-induced bifurcations hold for the general model. We do not attempt a methodical examination of how different model parameterizations might affect the numerical observations of section 4 . However, as noted in Section 2, the method of modeling consumer biomass conversion has been a point of considerable discussion in the literature, so is deserving of special attention. To this end, we have computed the analogous two-parameter bifurcation diagram Figure 4.1, replacing the default biomass conversion factor with the alternatives pictured in Figure A.1.

Short of presenting the full details, we simply report that our observations indicate that the qualitative characteristics of energy enrichment-induced bifurcation structure are not significantly impacted by the selection of $\mu_{c}(l)$. Figure 5.1 illustrates this point with a simultaneous plot of the two-parameter ( $b$ vs. $N_{T}$ ) diagrams for the Michaelis-Menten lower bound and Liebig upper bound for $\mu_{c}$, along with the default selection $\mu_{c}(l)=\left(1+l^{-1}-(1+l)^{-1}\right)^{-1}$. For the sake of clarity, transcritical bifurcation curves and the Hopf, homoclinic, saddlenode bifurcation curves are pictured separately. While the algebraic form of $\mu_{c}$ can impact the 
quantitative details of the diagrams, all choices share the same qualitative characteristics. The intermediate loss of periodic coexistence state via homoclinic bifurcations (see Figure 4.3) is most pronounced in the Liebig case, and is least evident in the Michaelis-Menten case. Examination of the lower panel with $N_{T} \approx .021$ shows that the Liebig case supports a $S N H C$ loss of periodic state, followed by a homoclinic re-creation of a period state, which is then lost in a Hopf bifurcation. In this case the model shares the essential bifurcation characteristics of both the LKE and Diehl models.

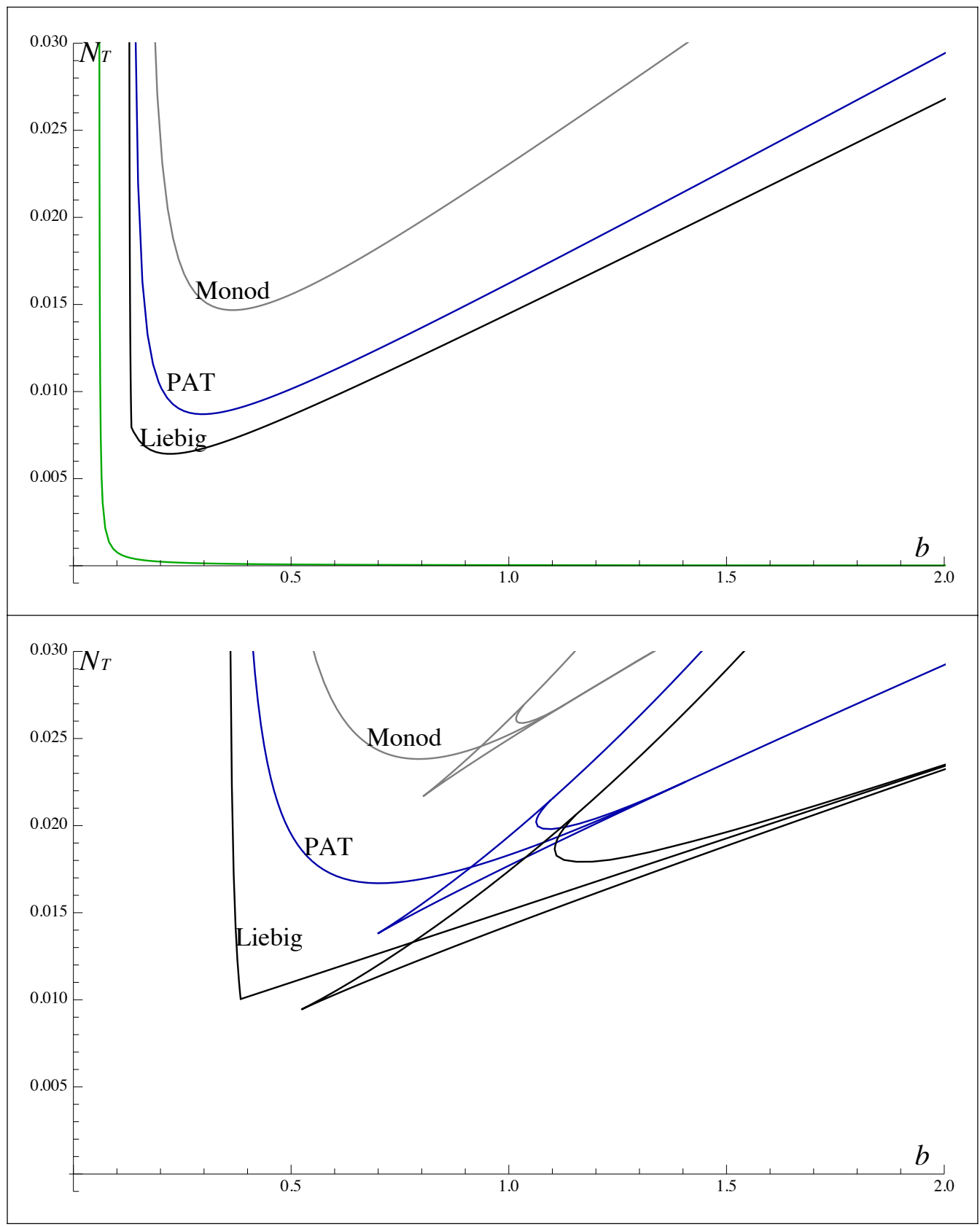


Figure 5.1: Simultaneous ( $b$ vs. $N_{T}$ ) bifurcation diagrams for smoothed Liebig $\mu_{c}(l)=\min [1, l]$ (black), "Poisson Arrival Time" (PAT) default $\mu_{c}(l)=\left(1+l^{-1}-(1+l)^{-1}\right)^{-1}$ (blue), and Michaelis-Menten (Monod) $\mu_{c}(l)=l /(1+l)$ (grey) consumer biomass conversion functions. For the purpose of using the AUTO [10] software, the Liebig case was smoothed using the aggregate $\tilde{\mu}_{c}(l)$ with $a=.001$; see Appendix A.1 for details. The top figure shows the producer transcritical bifurcation curve (equal in all three cases, and comparable to that of Figure 3.1) in green, and the coexistence transcritical bifurcation curves. The bottom figure shows the Hopf bifurcation, homoclinic bifurcation and saddlenode bifurcation curves. As usual, all parameters are the defaults of Table 4.1 .

These numerical results demonstrate that the selection of consumer biomass factor $\mu_{c}(l)$ can have a significant quantitative impact on the model's response to energy enrichment. For example, the "Poisson Arrival Time" (PAT) selection supports the same basic bifurcation structure as the Liebig case, but at (roughly) double the system nutrient and light enrichment levels. This quantitative difference provides a possible explanation of the different enrichment-induced bifurcation behaviors reported in [31] and [8]. The LKE study assumes the Liebig case and high system nutrient levels, both properties promoting the loss of periodic coexistence state in a Saddle-Node Homoclinic bifurcation. In contrast, the Diehl study employs the PAT consumer biomass conversion factor along with lower total system nutrient levels. These selections are more likely to cause the periodic coexistence solution to be lost in a Hopf bifurcation, with a corresponding re-stabilization of the high food quality coexistence steady state.

\section{Model Generalizations}

The general, process-based, construction of model $(2.1,2.2,2.3)$ is useful in that it helps to identify assumptions sometimes made implicitly in specific, more empirically-based, models. Modular mechanistic models also serve as steppingstones towards articulating various model generalizations. For example, nutrient released through producer and/or consumer mortality are here assumed to be immediately available for producer uptake. More realistically, one can introduce an intermediate nutrient sediment class that accepts producer/consumer detritus and releases mineralized nutrients suitable for producer use. See a producer monoculture system with sediment class in Diehl [7], and [8] for the analogous producer-consumer model. Alternately, the mineralized nutrient pool (here computed as nutrient not sequestered by the producer and consumer classes) could be opened, allowing a nutrient input flux and output loss. See [2], [19] and the references therein for related open systems, where system dynamics is often studied as a function of nutrient input concentration and system dilution (removal) rate.

The model of consumer functional response is here assumed to be independent of the stoichiometric characteristics of the producer population. However, in [34], [42] it has been observed that the nutrient status of the producer class can affect their rate 
of capture, as well as the associated consumer handling time and assimilation respiration. No attempts have been made here to consider such effects.

Regarding consumer use of producer nutrients, it has been assumed here that both producer structural and reserve nutrients are equally useful to consumer biomass creation, with common nutrient use efficiency $\varepsilon_{p}$. More realistically, the efficiencies for structural and reserve producer nutrients are likely to be different. Generalizing the derivation of the Appendix A.1, one could introduce distinct structural and reserve efficiencies, $\varepsilon_{p}^{S}$ and $\varepsilon_{p}^{R}$, respectively. The nutrient flux to the consumer synthesizing unit becomes $r_{N}=\left(\varepsilon_{p}^{S} q_{p}+\varepsilon_{p}^{R} R\right) f(P) P$, the consumer growth limitation index reads $l_{c}=\left(\varepsilon_{p}^{S} q_{p}+\varepsilon_{p}^{R} R\right) /\left(\delta_{c} q_{c}\right)$ and the consumer biomass conversion coefficient in equation (2.1) becomes $\delta_{c} \mu_{c}\left(\frac{\left(\varepsilon_{p}^{S} q_{p}+\varepsilon_{p}^{R} R\right)}{\delta_{c} q_{c}}\right)$. See the related "simplified DEB" model of [46] for an instance where $\varepsilon_{p}^{S}>0$, but $\varepsilon_{p}^{R}=0$.

Considering the overall derivation of (2.1-2.3), a more careful treatment of producer and consumer maintenance respiration would be of use. In the current model, maintenance respiration is combined with assimilation processes, and simplistically manifests itself in the model through the carbon use efficiency coefficients, $\delta_{p}$ and $\delta_{c}$. More realistically, maintenance respirations could be modeled as a function of producer and consumer biomass. A producer respiration carbon loss term similar to the current mortality term could be added to (2.1), although maintenance respiration is not likely to affect the producer nutrient pool, $N_{p}$. Given our modeling assumption of constant nutrient: carbon stoichiometry, $q_{c}$, adding a similar "mortality-like" respiration term to (2.1) leads to issues regarding the fate of the associated consumer nutrient. See [2], [19], [3], [26] and the references therein for related approaches to modeling maintenance respiration.

\section{Conclusions}

In recent years a number of authors have contributed to the understanding of producer consumer systems in which the producer's nutrient content is assumed to be variable. We have presented here a general model whose construction is based on general, qualitative assumptions, rather than relying on specific algebraic forms for model components. In particular, the dependence of producer primary productivity and consumer biomass conversion on producer nutrient content here is motivated by a generalized use of the synthesizing unit concept. This approach forces one to focus on system characteristics that are independent of the algebraic details of how nutrient constraints are described in the model. Despite the generality of our model, we have shown that the associated monoculture (producer only) system is tractable. Numerical experiments indicate that the details of how producer nutrient stores affect its primary productivity have little effect on the associated consumer-free system. 
We have parameterized (Table 4.1) the general model to the context of algae-Daphnia systems, and for this realization numerical simulations demonstrate the diversity of energy enrichment-induced dynamic changes that can be observed at different system nutrient levels. Under modest nutrient levels, energy enrichment can result in a locally attracting (sometimes periodic) coexistence state simultaneous to a locally attracting low food quality equilibrium state. Further enrichment may induce a loss of periodic state in a variety of different bifurcation scenarios. In particular, largeamplitude periodic coexistence solutions may intermittently re-appear under additional energy enrichment. Our model shares many of the essential modeling assumptions of both the LKE [31] and Diehl [8] stoichiometric producer-consumer models. Our results reconcile these two studies in that our model exhibits for high system nutrient levels the energy enrichment responses of LKE, while for low nutrient levels it supports the responses of the Diehl model. Furthermore, our numerical bifurcation computations indicate that the algebraic form assumed of the consumer biomass conversion submodel has little qualitative effect on the overall bifurcation structure of the model. However, the specifics of consumer biomass conversion can cause observable quantitative differences on the model's bifurcation structure.

Acknowledgments: The authors thank the referees for their suggestions.

\section{Appendix A: Stoichiometrically-Constrained Biomass Production}

A.1 Consumer Biomass Production. The conceptual basis of the biomass Synthesizing Unit [25] is that of a hypothetical production site that accepts simultaneous fluxes of substrates, and generates stoichiometrically-constrained biomass. We assume that the consumer SU receives per capita fluxes of carbon with mean rate $r_{C}=\delta_{c} f(P) P$ and nutrient with mean rate $r_{N}=\varepsilon_{c}\left(q_{p}+R\right) f(P) P$, both proportionate to the per capita predation rate. The parameters $\delta_{c}<1$ and $\varepsilon_{c}<1$ represent respective reductions of ingested carbon and nutrients due to egestion, digestive processes and respiration.

Following [25], one hypothesizes substrate arrival probabilities $\boldsymbol{P}_{e}, \boldsymbol{P}_{n}$ for the arriving carbon and nutrient streams. If the probabilities are normalized to have mean arrival times one, the mean effective substrate arrival time is given by $\int_{0}^{\infty} 1-P_{e}\left(r_{C} u\right) P_{n}\left(\frac{r_{N}}{q_{C}} u\right) d u$. Assuming that there is a negligible production period in assembling arrived substrates, the specific (per capita) consumer biomass growth rate is given by

$$
1 / \int_{0}^{\infty} 1-\boldsymbol{P}_{e}\left(r_{C} u\right) \boldsymbol{P}_{n}\left(\frac{r_{N}}{q_{c}} u\right) d u=r_{C} / \int_{0}^{\infty} 1-\boldsymbol{P}_{e}(v) \boldsymbol{P}_{n}\left(l_{c} v\right) d v=\delta_{c} \mu_{c}\left(l_{c}\right) f(P) P
$$


with $\mu_{c}(l) \equiv 1 / \int_{0}^{\infty} 1-\boldsymbol{P}_{e}(v) \boldsymbol{P}_{n}(l v) d v$ and $l_{c}=\left(\varepsilon_{c}\left(q_{p}+R\right) / \delta_{c}\right) / q_{c}$. Based on this derivation, $l_{c}$ represents the ratio of the nutrient and carbon fluxes arriving at the SU, in relation to the stoichiometric requirements of consumer biomass. One can view $l_{c}<1$ to be an indication of nutrient-poor food, while $l_{c}>1$ corresponds to food containing sufficient nutrient content to permit carbon-limited biomass creation. The reciprocal of $l_{c}$ corresponds to the "limiting coefficient" defined in [35]. The function $\mu_{c}(l)$ can be thought of as a reduction factor due to the stoichiometric differences between the processed food stream and consumer biomass. Our technical assumptions on $\mu_{c}(l)$ are based on this construction.

Proposition A.1. If $\boldsymbol{P}_{e}$ and $\boldsymbol{P}_{n}$ are functions each satisfying $0 \leq \boldsymbol{P}(v) \leq 1, \lim _{v \rightarrow \infty} \boldsymbol{P}(v)=1$, $\mathcal{P}$ non-decreasing, and $\int_{0}^{\infty} 1-\mathcal{P}(v) d v=1$, then $\mu(l) \equiv 1 / \int_{0}^{\infty} 1-\mathcal{P}_{e}(v) \boldsymbol{P}_{n}(l v) d v$ is increasing in $l$, with $l /(1+l) \leq \mu_{c}(l) \leq \min (1, l)$. Moreover, for $l>0$, both $\mu(l) / l$ and $\frac{d \mu}{d l}$ are non-increasing in $l$.

Proof: Because $\boldsymbol{P}_{n}\left(l_{c} v\right)$ is increasing in $l$, it follows easily that $1 / \mu(l)$ is decreasing in $l$. Under our assumptions on $\boldsymbol{P}_{e}(v)$ and $\boldsymbol{P}_{n}(v)$, we have $1 \leq\left(1-\boldsymbol{P}_{e}(v)\right)\left(1-\boldsymbol{P}_{n}(l v)\right)$. Expanding and rearranging this inequality, one gets $1 / \mu(l)=\int_{0}^{\infty} 1-\boldsymbol{P}_{e}(v) \boldsymbol{P}_{n}(l v) d v \leq \int_{0}^{\infty} 1-\boldsymbol{P}_{e}(v) d v+\int_{0}^{\infty} 1-\boldsymbol{P}_{n}(l v) d v=1+1 / l$ and the stated lower bound on $\mu(l)$. The upper bound on $\mu(l)$ follows from the observation that since $\boldsymbol{P}_{e}(v), \boldsymbol{P}_{n}(l v) \leq 1$, both $1 / \mu(l) \geq \int_{0}^{\infty} 1-\boldsymbol{P}_{e}(v) d v$ (which equals 1 ) and $1 / \mu(l) \geq \int_{0}^{\infty} 1-P_{n}(l v) d v$ (which equals $1 / l$.) Thus, $1 / \mu(l) \geq \max (1,1 / l)$ (which equals $1 / \min (1, l)$.) By change of variables in the integral, one gets $l / \mu(l)=\int_{0}^{\infty} 1-\boldsymbol{P}_{e}\left(\frac{s}{l}\right) \boldsymbol{P}_{n}(s) d s$. Thus $l / \mu(l)$ is non-decreasing in $l$ since $\boldsymbol{P}_{e}$ is nondecreasing. In fact, if we express $P_{e}(u)=\int_{0}^{u} k_{e}(s) d s$, then $\frac{d}{d l}(l / \mu(l))=\frac{1}{l^{2}} \int_{0}^{\infty} k_{e}(s / l) \boldsymbol{P}_{n}(s) s d s \geq 0$. Finally, if we further express $\boldsymbol{P}_{n}(u)=\int_{0}^{u} k_{n}(s) d s$, then differentiation of $\mu^{-1}(l)$ results in $\mu^{-2} \frac{d \mu}{d l}=\int_{0}^{\infty} P_{e}(u) k_{n}(l u) u d u$. It follows that 
$\frac{d \mu}{d l}=\left(\frac{\mu(l)}{l}\right)^{2} \int_{0}^{\infty} P_{e}(s / l) k_{n}(s) s d s \geq 0$, from which $\frac{d \mu}{d l}$ is seen to be non-increasing in $l$ because $P_{e}$ and $l / \mu(l)$ are non-decreasing.

If the arrival rates for the carbon and nutrient fluxes are both assumed constant then $\boldsymbol{P}_{e}(v)=\boldsymbol{P}_{n}(v)=0$ if $v<1$, and $\boldsymbol{P}_{e}(v)=\boldsymbol{P}_{n}(v)=1$ if $v>1$. One obtains $\mu_{c}(l)=\min [1, l]$, the Liebig "Law of the Minimum." In this case, $\delta_{c} \mu_{c}\left(l_{c}\right)=\min \left[\delta_{c}, \varepsilon_{c}\left(q_{p}+R\right) / q_{c}\right]$, as discussed in [20]. This further reduces to $\delta_{c} \min \left[1,\left(q_{p}+R\right) / q_{c}\right]$ under the assumption of equal assimilation efficiencies for carbon and nutrient. See [31], [27] and [46]. However, many authors have observed that nutrient assimilation efficiency is often higher than carbon assimilation efficiency. See [17], [36], and [5].

The Liebig Law has been criticized for the lack of smoothness it introduces into the consumer biomass production term in (2.1) ([39], [35], [26], [28]). The resulting discontinuity in the associated linearized system introduces difficulties for numerical bifurcation software [10]. Hessen and Bjerkeng [20] discuss an empirical smoothing of the Liebig case. As shown above, the Liebig case may be viewed as the (perhaps atypical) upper extreme for SU-based biomass conversion factors, with more mechanically realistic alternatives producing lower biomass conversion rates.

Many different biomass conversion efficiency functions can be obtained by taking different forms for $\boldsymbol{P}_{e}$ and $\boldsymbol{P}_{n}$. For example, Kooijman's original work considers the case where $\boldsymbol{P}_{e}$ and $\boldsymbol{P}_{n}$ are defined in terms of Poisson and Erlangian distributions. However, for these the algebraic form of $\mu_{c}(l)$ becomes prohibitive in all but the Poisson case $\boldsymbol{P}_{e}(v)=\boldsymbol{P}_{n}(v)=1-e^{-v}$, where $\mu_{c}(l)=\left(1+l^{-1}-(1+l)^{-1}\right)^{-1}$. This case was originally derived in [39], where it (there called the "Poisson Arrival Time" model) was shown to compare favorably to other nutrient limitation models in its ability to model nutrient-limited growth in both chemostat and terrestrial data sets.

Intermediate to this and the Liebig case, one could consider the situation where nutrients are assumed to arrive to the SU as a Poisson process $P_{n}(v)=1-e^{-v}$ but carbon arrives at a constant rate, as in the Liebig case. The resulting biomass conversion factor then is easily computed to be $\mu_{c}(l)=l /\left(e^{-l}+l\right)$. For constant carbon arrival rate, but with nutrient arrival described in terms of the gamma distributions $\boldsymbol{P}_{n}(v)=1-(1+2 v) e^{-2 v}$ and $\boldsymbol{P}_{n}(v)=1-\left(1+3 v+\frac{1}{2}(3 v)^{2}\right) e^{-3 v}$ one gets $\mu_{c}(l)=l /\left((1+l) e^{-2 l}+l\right)$ and $\mu_{c}(l)=l /\left(\left(1+2 l+\frac{3}{2} l^{2}\right) e^{-3 l}+l\right)$, respectively. One can show that the Michaelis-Menten $l /(1+l)$ lower bound on $\mu_{c}(l)$ is sharp in the sense that there exist arrival probabilities for which the resulting $\mu_{c}(l)$ approximates $l /(1+l)$ arbitrarily closely. Specifically, for $P_{e}(v)=1-e^{-v}$ and the family $\boldsymbol{P}_{n}^{(\tau)}(v)=\{1-\tau$ if $v<1 / \tau, 1$ if $v>1 / \tau\}$ defined for parameter $0<\tau \leq 1$, one can compute that $\mu_{c}^{(\tau)}(l)=l /\left(1+l+\tau l\left(e^{-1 / \tau l}-1\right)\right) \rightarrow l /(1+l)$ as $\tau \rightarrow 0$. 
The primary advantage of this generalized SU-based construction lies in its processmotivated framework for describing nutrient-regulated biomass conversion. The Liebig "Law of the Minimum" is conceptually simple, but attributes to the whole consumer population an identical, simultaneous response to the nutrient content of its food source. More realistically, nutrient quality will vary among members of the producer population, resulting in an aggregated version of the Liebig term. This can be made analytically rigorous using the "transmutation" concept discussed in [38]. Specifically, if one assumes that the consumer limitation index is distributed about its mean with probability distribution $\eta$ (which we take to have support on the interval $[-a, a], a<1$ ), then the expected biomass conversion efficiency for a population of "Liebig-type" individuals is given by $\tilde{\mu}_{c}(l)=\int_{-a}^{a} \min [l+u, 1] \eta(u) d u$. One can show that regardless of the choice of distribution function, $\tilde{\mu}_{c}(l)=l$ for $l \leq 1-a, \tilde{\mu}_{c}(l)=1$ for $l \geq 1+a$, and that $\tilde{\mu}_{c}(l)$ is continuously differentiable in $l$. In the simplest case, where $\eta(u)=1 / 2 a$ on its support, one calculates that for $1-a \leq l \leq 1+a$, $\tilde{\mu}_{c}(l)=l-(l-1+a)^{2} / 4 a$.

Figure A.1 provides a comparison of some of the biomass conversion factors that we have discussed, including $\mu_{c}^{(1 / 4)}(l)$ and the aggregate $\tilde{\mu}_{c}(l)$ for $a=1 / 3$.



Figure A.1: Alternate consumer biomass conversion factors $\mu_{c}(l)$. From top to bottom: $\min [1, l], \tilde{\mu}_{c}(l)$ with $a=1 / 3, l /\left(\left(1+2 l+\frac{3}{2} l^{2}\right) e^{-3 l}+l\right), l /\left((1+l) e^{-2 l}+l\right)$, $l /\left(e^{-l}+l\right),\left(1+l^{-1}-(1+l)^{-1}\right)^{-1}, l /\left(1+l+\frac{l}{4}\left(e^{-4 / l}-1\right)\right)$ and greatest lower bound 
$l /(1+l)$.

A.2 Producer Biomass Production. We assume that producer SU receives a carbon flux $r_{C}=\delta_{p} g(P) P$, and a nutrient flux $r_{N}=\varepsilon_{p} R g(P) P$ whose density is determined by the concentration of producer nutrient reserves, $R$. Parameters $0 \leq \delta_{p}, \varepsilon_{p} \leq 1$ represent reductions of carbon and nutrient fluxes due to maintenance and growth respiration. Here interpreting $\boldsymbol{P}_{e}, \boldsymbol{P}_{n}$ to be the substrate arrival probabilities for the producer SUs, one computes the expected substrate arrival rate as $\int_{0}^{\infty} 1-\mathcal{P}_{e}\left(r_{C} u\right) \boldsymbol{P}_{n}\left(\frac{r_{N}}{q_{C}} u\right) d u$. Analogous to the consumer case, the specific producer biomass growth rate is given by $\delta_{p} \mu_{p}\left(l_{p}\right) g(P) P$ with $\mu_{p}(l) \equiv 1 / \int_{0}^{\infty} 1-\mathcal{P}_{e}(v) \boldsymbol{P}_{n}(l v) d v$ and $l_{p}=r_{N} / q_{p} r_{C}=\varepsilon_{p} R / \delta_{p} q_{p}$.

\section{Appendix B: Model Analysis}

B.1 Well-Posedness of the Model. By standard estimates, solutions of (2.1-2.3) with non-negative initial conditions will remain so for $t>0$. Using the properties of $\mu_{p}$, for small values of $R$ the term in (2.4) $\delta_{p} \mu_{p}\left(l_{p}\right)\left(R+q_{p}\right) \simeq \delta_{p} l_{p} q_{p}$, which equals $\varepsilon_{p} R$ if $q_{p}>0$. If $q_{p}=0$ then $\mu_{p} \equiv 1$ and $\delta_{p} \mu_{p}\left(l_{p}\right)\left(R+q_{p}\right)=\delta_{p} R$. In either case, (2.4) shows that the nutrient reserve concentration $R(t)$ also remains non-negative for $t>0$. The following result verifies that the total amount of nutrient sequestered by the producer and consumer remains less than the total system nutrient pool.

Proposition B.1 If $N_{p}(0)+q_{c} C(0)<N_{T}$, then $N_{p}(t)+q_{c} C(t)<N_{T}$ for all $t \geq 0$. Using $N_{p}=\left(R+q_{p}\right) P$, this inequality results in the bound on the producer's nutrient reserve concentration $R<\left(N_{T}-q_{c} C-q_{p} P\right) / P$.

Proof: Let $t$ denote the smallest value for which $N_{p}(t)+q_{c} C(t)=N_{T}$. Then at such $t$,

$$
\begin{aligned}
N_{p}^{\prime}+q_{c} C^{\prime} & =h\left(N_{m}\right) g(P) P-d_{p}\left(R+q_{p}\right) P-\left(R+q_{p}\right) f(P) P C+q_{c}\left[\delta_{c} \mu_{c}\left(\frac{\varepsilon_{c}}{\delta_{c}} \frac{R+q_{p}}{q_{c}}\right) f(P) P-d_{c}\right] C \\
& =h\left(N_{T}-N_{p}-q_{c} C\right) g(P) P+\left[-\left(R+q_{p}\right)+q_{c} \delta_{c} \mu_{c}\left(\frac{\varepsilon_{c}}{\delta_{c}} \frac{R+q_{p}}{q_{c}}\right)\right] P f(P) C-d_{p}\left(R+q_{p}\right) P-q_{c} d_{c} C \\
& \leq h(0) g(P) P+\left[-\left(R+q_{p}\right)+\varepsilon_{c}\left(R+q_{p}\right)\right] P f(P) C-d_{p}\left(R+q_{p}\right) P-q_{c} d_{c} C
\end{aligned}
$$

since $\mu_{c}(l) \leq l$. Using $h(0)=0$ and $\varepsilon_{p} \leq 1$, one gets $N_{p}^{\prime}+q_{c} C^{\prime} \leq-\min \left[d_{c}, d_{p}\right]\left(N_{p}+q_{c} C\right)=-\min \left[d_{c}, d_{p}\right] N_{T}<0$. This is a contradiction.

B.2 Persistence in the Monoculture System. The monoculture case $(C \equiv 0)$ is described by the equations 


$$
\begin{aligned}
& P^{\prime}=\left[\delta_{p} \mu_{p}\left(\varepsilon_{p} R / \delta_{p} q_{p}\right) g(P)-d_{p}\right] P \\
& R^{\prime}=g(P)\left[h\left(N_{T}-q_{p} P-R P\right)-\delta_{p} \mu_{p}\left(\varepsilon_{p} R / \delta_{p} q_{p}\right)\left(R+q_{p}\right)\right]
\end{aligned}
$$

For the case where the producer productivity is not nutrient-dependent $\left(q_{p}=0, \mu_{p} \equiv 1\right)$ this system simplifies to the uncoupled pair $P^{\prime}=\left[\delta_{p} g(P)-d_{p}\right] P$, $R^{\prime}=g(P)\left[h\left(N_{T}-R P\right)-\delta_{p} R\right]$. Under the assumptions of $g$ there is a unique nontrivial monoculture equilibrium $P=\bar{P}^{m}>0$ to the first equation for when $\delta_{p} g(0) \geq d_{p}$. Under our assumptions on $h$, the expression $h\left(N_{T}-R \bar{P}^{m}\right)-\delta_{p} R$ is strictly decreasing in $R$, and has a unique zero $R=\bar{R}^{m}$ on the interval $0<R<N_{T} / \bar{P}^{m}$. It is easily shown that $\left(\bar{P}^{m}, \bar{R}^{m}\right)$ is globally attracting to all solutions with $P>0, R>0$.

For the case $q_{p}>0$ we first describe the $P^{\prime}=0$ nullcline for (B.1). Define $\tilde{P}>0$ to be the unique solution of $\delta_{p} g(\tilde{P})=d_{p}$. For each $0<P<\tilde{P}$, one has $\delta_{p} g(P)>d_{p}$. By the conditions on $\mu_{p}$ there is a unique value $R=R_{p}(P)>0$ satisfying $\left.\delta_{p} \mu_{p} \varepsilon_{p} R_{p}(P) / \delta_{p} q_{p}\right)=d_{p} / g(P)$. As a function of $P$, nullcline $R_{p}(P)$ is continuous and is increasing in $P$, with $\mu_{P}\left(\varepsilon_{p} R_{p}(P) / \delta_{p} q_{p}\right) \rightarrow 1$ as $P \rightarrow \tilde{P}$. When $P=0, R=R_{p}(0)$ satisfies $g(0)=d_{p} / \delta_{p} \mu_{p}\left(\varepsilon_{p} R / \delta_{p} q_{p}\right)$.

The $R^{\prime}=0$ nullcline for (B.2) satisfies

$$
h\left(N_{T}-q_{p} P-R P\right)=\delta_{p} \mu_{p}\left(\varepsilon_{p} R / \delta_{p} q_{p}\right)\left(R+q_{p}\right) .
$$

For fixed $P, 0<P<N_{T} / q_{p}$ the left side of (B.3) defines a non-increasing function of $R$ on the interval $0 \leq R \leq\left(N_{T}-q_{p} P\right) / P$ that is positive at $R=0$ and vanishes at $R=\left(N_{T}-q_{p} P\right) / P$. By our assumptions on $\mu_{p}$, the right side of (B.3) defines an increasing function of $R$ that vanishes at $R=0$, and is positive at $R=\left(N_{T}-q_{P} P\right) / P$. Therefore, there is a unique solution $R=R_{R}(P)>0$ of (B.3). As a function of $P$, the nullcline $R=R_{R}(P)$ is continuous and non-increasing in $P$. When $P=0, R_{R}(0)=R_{\max }$ is implicitly defined as the unique solution to $h\left(N_{T}\right)=\delta_{p} \mu_{p}\left(\varepsilon_{p} R_{\max } / \delta_{p} q_{p}\right)\left(R_{\max }+q_{p}\right)$. By the monotonicities of $R_{p}(P)$ and $R_{R}(P)$, there will exist an equilibrium $(P, R)=\left(\bar{P}^{m}, R_{R}\left(\bar{P}^{m}\right)\right)$ (which is unique) with $P>0$ and $R>0$ if and only if $R_{P}(0)<R_{R}(0)$. This is equivalent to the assumptions of the theorem. 


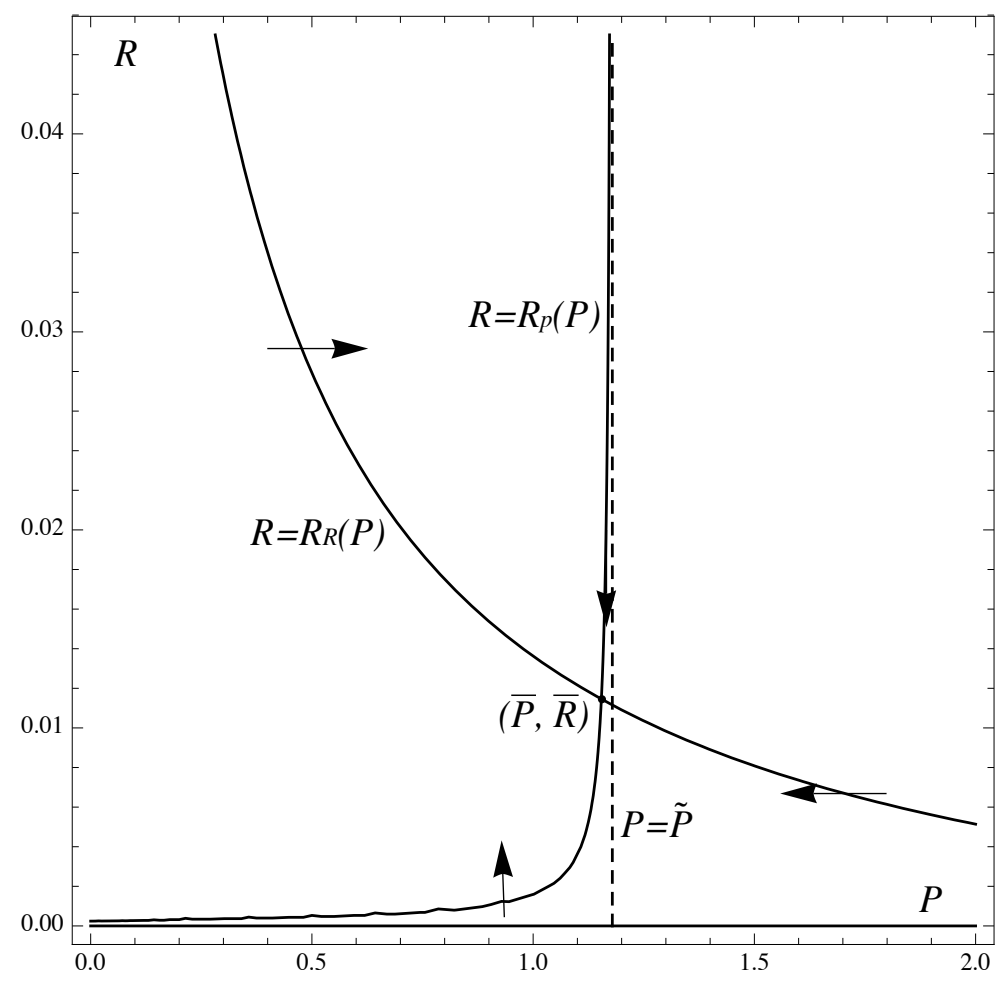

Figure B.1. A typical phase plane for the monoculture system, illustrating system nullclines and the equilibrium $(\bar{P}, \bar{R})=\left(\bar{P}^{m}, \bar{R}^{m}\right)$. The case shown assumes $g(P)=\max (r[b-P / k], 0), \mu_{p}(l)=l /(1+l), l=\frac{\varepsilon_{p}}{\delta_{p}} \frac{R}{q_{p}}, h(n)=\beta n$, and parameters $r=1.1, b=1 ., k=1.25, \varepsilon_{p}=.9, \delta_{p}=.8, q_{p}=.0045, \beta=6.0$, and $N_{T}=.02$. See Section 4 for a discussion of parameter selections and units.

To prove that equilibrium is globally attracting, we multiply the right sides of (B.1, B.2) by the Dulac function $(P g(P))^{-1}$, and compute $\frac{\partial}{\partial P}\left(\delta_{p} \mu_{p}\left(\frac{\varepsilon_{p}}{\delta_{p}} \frac{R}{q_{p}}\right)-d_{p} / g(P)\right)+\frac{\partial}{\partial R}\left(h\left(N_{T}-q_{p} P-R P\right) / P-\delta_{p} \mu_{p}\left(\varepsilon_{p} R / \delta_{p} q_{p}\right)\left(R+q_{p}\right) / P\right) \leq 0$. Dulac's Criterion rules out the existence of closed cycles, and global stability follows from a standard application of Poincare-Bendixson theory.

\section{References}

[1] Abrams, P. A. and J. Roth, 1994. The Responses of Unstable Food Chains to Enrichment, Evolutionary Ecology 8, 150-171.

[2] Andersen, T., Pelagic Nutrient Cycles: Herbivores as Sources and Sinks, Ecological Studies 129, Springer, Berlin, 1997. 
[3] Andersen, T., J. Elser and D. Hessen, 2004. Stoichiometry and Population Dynamics, Ecology Letters 7, 884-900.

[4] Butler, G. J. and G. S. K. Wolkowicz, 1985. A Mathematical Model of the Chemostat with a General Class of Functions Describing Nutrient Uptake, SIAM J. Appl. Math. 45 (1), 138-151.

[5] DeMott, W. R., R. D. Gulati and K. Siewertsen, 1998. Effects of PhosphorousDeficient Diets on the Carbon and Phosphorous Balance of Daphnia magna, Limnol. Oceanogr. 43 (6), 1147-1161.

[6] Diehl, S., 2002. Phytoplankton, Light, and Nutrients in a Gradient of Mixing Depths: Theory, Ecology 83 (2), 386-398.

[7] Diehl, S., S. Berger, and R. Wohrl, 2005. Flexible Nutrient Stoichometry Mediates Environmental Influences on Phytoplankton and its Resources, Ecology 86 (11), 2931-2945.

[8] Diehl, S., 2007. Paradoxes of Enrichment: Effects of Increased Light versus Nutrient Supply on Pelagic Producer-Grazer Systems, The American Naturalist 169 (6), E173-E191.

[9] Droop, M., 1974. The Nutrient Status of Algal Cells in Continuous Culture, J. Mar. Biol. Ass. U. K. 54, 825-855.

[10] Doedel, E. J., A. Champneys, T. Fairgrieve, Y. Kuznetsov, B. Sandstede and X. Wang, 1997. AUTO 97: Continuation and Bifurcation Software for Ordinary Differential Equations. Technical Report. Concordia University, Montreal, Quebec, Canada.

[11] Elser, J., W. Fagan, R. Denno, D. Dobberfuh, A. Folarin, A. Huberty, S. Interlandi, S. S. Kilham, E. McCauley, K. Schulz, E. Siemann and R. Sterner, 2000. Nutritional Constraints in Terrestrial and Freshwater Food Webs, Nature 408 (30), 578-580.

[12] Fan, M., I. Loladze, Y. Kuang, and J. Elser, 2005. Dynamics of a Stoichiometric Discrete Producer-Grazer Model, Journal of Difference Equations and Applications 11 (4-5) 347-364.

[13] Freedman, H. I. and G. S. K. Wolkowicz, 1986. Predator-Prey Systems with Group Defense: The Paradox of Enrichment Revisited, Bull. Math. Biol. 48, 493-508.

[14] Grover, J. P., 1989. Phosphorus-dependent growth kinetics of 11 species of freshwater algae, Limnol. Oceanogr. 34 (2), 341-348. 
[15] Grover, J. P., 1991. Resource Competition in a Variable Environment: Phytoplankton Growth According to the Variable-Internal Stores Model, The American Naturalist 138 (4), 811-835.

[16] Grover, J. P., 2002. Stoichiometry, Herbivory and Competition for Nutrients: Simple Models Based on Planktonic Ecopsystems, Journal of Theoretical Biology 214, 599-618.

[17] Grover, J. P., 2003. The Impact of Variable Stoichiometry on Pedator-Prey Interactions: A Multinutrient Approach, The American Naturalist 162 (1), 29-43.

[18] Grover, J. P. and R. D. Holt, 1998. Disentangling Resource and Apparent Competition: Realistic Models for Plant-herbivore Communities, J. Theoretical Biology 191, 353-376.

[19] Hanegraaf, P. P. F. and B. W. Kooi, 2002. The Dynamics of a Tri-trophic Food Chain with Two-Component Populations from a Biochemical Perspective, Ecological Modelling 152, 47-64.

[20] Hessen, D. O. and B. Bjerkeng, 1997. A Model Approach to Planktonic Stoichiometry and Consumer-Resource Stability, Freshwater Biology 38, 447-471.

[21] Huisman, J. and F. Weissing, 1994. Light-Limited Growth and Competition for Light in Well-mixed Aquatic Environments: An Elementary Model, Ecology 75 (2), 507-520.

[22] Huisman, J. and F. Weissing, 1995. Competition for Nutrients and Light in a Mixed Water Column: A Theoretical Analysis, The American Naturalist 146 (4), 536564.

[23] Ivlev, V. S., Experimental Ecology of the Feeding of Fishes, Yale University Press, New Haven, CT, 1961.

[24] Klausmeier, C., E. Litchman, and S. Levin, 2004. Phytoplankton Growth and Stoichiometry Under Multiple Nutrient Limitation. Limnol. Oceanogr. 49 (4, part 2), 1463-1470.

[25] Kooijman, S. A. L. M., 1998. The Synthesizing Unit as Model for the Stoichiometric Fusion and Branching of Metabolic Fluxes, Biophysical Chemistry 73, 179-188.

[26] Kooijman, S. A. L. M., T. Andersen, and B. W. Kooi, 2004. Dynamic Energy Budget Representations of Stoichiometric Constraints on Population Dynamics, Ecology 85 (5), 1230-1243.

[27] Kuang, Y., J. Huisman and J. Elser, 2004. Stoichiometric Plant-Herbivore Models and their Interpretation, Mathematical Biosciences and Engineering 1 (2), 215-222. 
[28] Kuijper, L., B. Kooi, T. Andersen, S. Kooijman, 2004. Stoichiometry and FoodChain Dynamics, Theoretical Population Biology 66, 323-339.

[29] Kuznetsov, Y., Elements of Applied Bifurcation Theory, Applied Mathematical Sciences 112, Springer, New York, 1998.

[30] Lin, H.-R. L., B. Peckham, H. Stech and J. Pastor, 2011. Enrichment in a Stoichiometric Model of Two Producers and One Consumer, Journal of Biological Dynamics, 1751-1766.

[31] Loladze, I., Y. Kuang and J. Elser, 2000. Stoichiometry in Producer-Grazer Systems: Linking Energy Flow and Element Cycling, Bulletin of Mathematical Biology $62,1137-1162$.

[32] Loladze, I., Y. Kuang, J. Elser, and W. Fagan, 2004. Theoretical Population Biology, Competition and Stoichiometry: Coexistence of Two Predators on One Prey 65, 1-15.

[33] Mathematica 7.0, Wolfram Research, Champaign, Illinois, 2008.

[34] McCauley, E., W. Murdoch and S. Watson, 1988. Simple Models and Variation in Plankton Densities Among Lakes, The American Naturalist 132 (3), 383-403.

[35] Muller, E., R. Nisbet, S. Kooijman, J. Elser, and E. McCauley, 2001. Stoichiometric Food Quality and Herbivore Dynamics, Ecology Letters 4, 519-529.

[36] Nisbet, R., M., E. McCauley, A. M. deRoos, W. W. Murdoch, W. S. C. Gurney, 1991. Population Dynamics and Element Recycling in and Aquatic Plant-Herbivore System, Theoretical Population Biology 40, 125-147.

[37] Norberg, J. and D. DeAngelis, 1997. Temperature Effects on Stocks and Stability of a Phytoplankton-Zooplankton Model and the Dependence on Light and Nutrients. Ecological Modelling 95, 75-861.

[38] O'Neill, R. V., 1979. Transmutation Across Hierarchical Levels. In: Systems Analysis of Ecosystems, G. S. Innis and R. V. O'Neill (Eds.), International Co-operative Publishing House, Fairland, MD, pp. 59-78.

[39] O’Neill, R. V., D. L. DeAngelis, J. J. Pastor, B. J. Jackson and W. M. Post, 1989. Multiple Nutrient Limitations in Ecological Models, Ecological Modeling 46, 147-163.

[40] Rosenzweig, M. L., 1971. Paradox of Enrichment: Destabilization of Exploitation Ecosystems in Ecological Time, Science, New Series 171 (3969), 385-387. 
[41] Rosenzweig, M. L., and R. H. MacArthur, 1963. Graphical Representation and Stability Conditions of Predator-Prey Interactions, The American Naturalist 97 (895), 209-223.

[42] Schatz, G. and E. McCauley, 2007. Foraging Behavior by Daphnia in Stoichiometric Gradients of Food Quality, Oecologia 153, 1021-1030.

[43] Stech, H. W., Peckham, B., and J. Pastor, Enrichment Effects in a Simple Stoichiometric Producer-Consumer Population Growth Model, submitted.

[44] Stech, H. W., Peckham, B., and J. Pastor, Quasi-equilibrium Reduction in a General Class of Stoichiometric Producer-Consumer Models, submitted.

[45] Stiefs, D., G. A. K. van Voorn, B. Kooi, U. Feudel and T. Gross, 2010. Food Quality in Producer-Grazer Models: A Generalized Analysis, The American Naturalist 176 (3), 367-380.

[46] Sui, G., M. Fan, I. Loladze and Y. Kuang, 2007. The Dynamics of a Stoichiometric Plant-Herbivore Model and its Discrete Analog, Mathematical Biosciences and Engineering 4 (1), 1-18.

[47] Wang, H., Y. Kuang and I. Loladze, 2008. Dynamics of a Mechanistically Derived Stoichiometric Producer-Grazer Model, Journal of Biological Dynamics 2 (3), 286-296.

[48] Wang, H., L. Smith, Y. Kuang and J. Elser, 2007. Dynamics of a Stoichiometric Bacteria-Grazer Interactions in the Epilimnion, SIAM J. Appl. Math. 68, 503-522.

[49] Wang, H., D., K. Dunning, J. Elser, Y. Kuang, 2009. Daphnia Species Invasion, Competitive Exclusion, and Chaotic Coexistence, Discrete and Continuous Dynamical Systems Series B 12 (2), 481-493.

[50] Weissing, F. and J. Huisman, 1994. Growth and Competition in a Light Gradient, Journal of Theoretical Biology 168, 323-336. 